
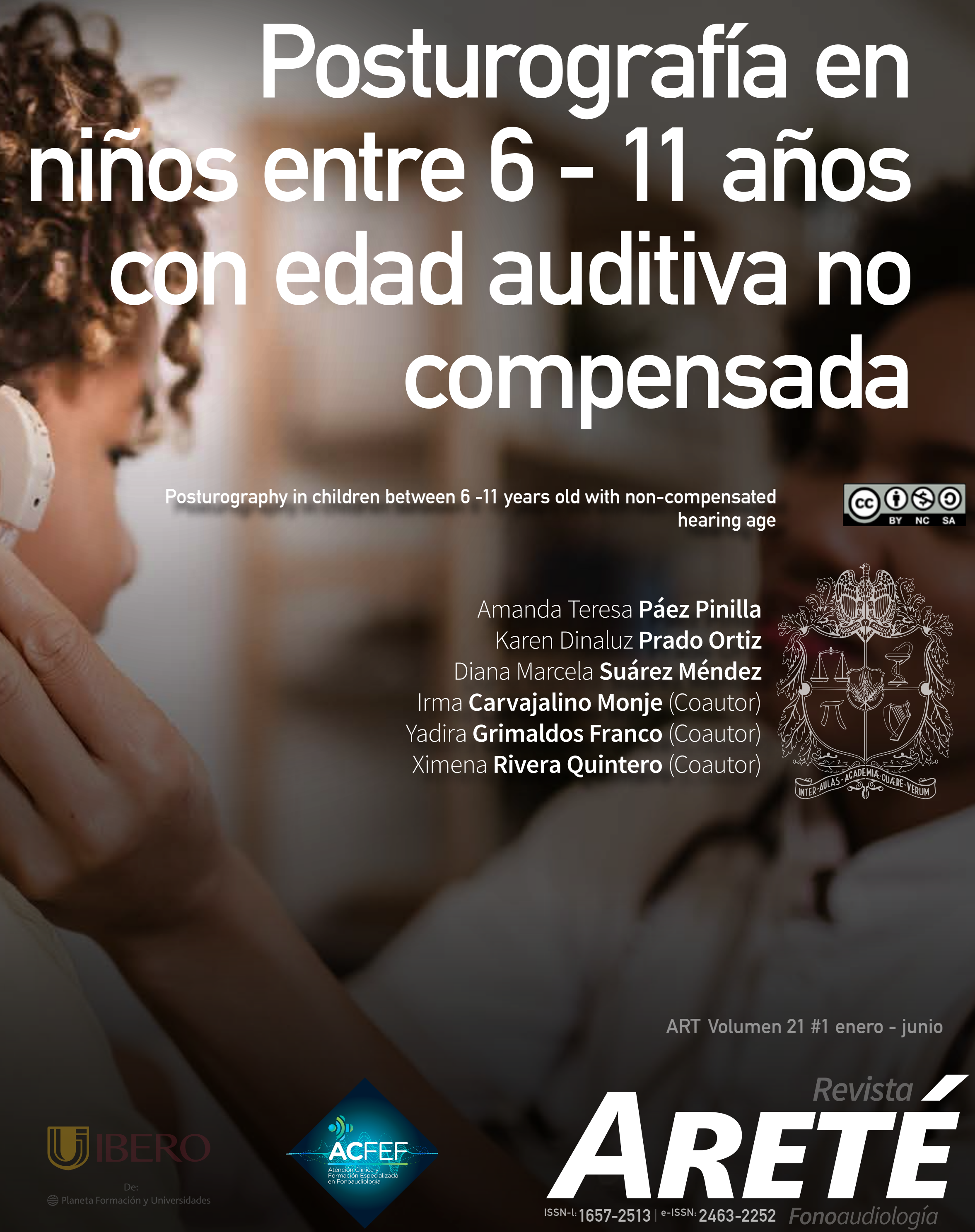


\section{ARETÉ \\ $1657-2513$}

ID:

10.33881/1657-2513.art.21101

Title: $\quad$ Posturography in children between 6 -11 years old with non-compensated hearing age

Título: $\quad$ Posturografía en niños entre 6 -11 años con edad auditiva no compensada

Alt Title / Título alternativo:

Posturography in children between 6 -11 years old with non-compensated hearing age

[es]: $\quad$ Posturografía en niños entre 6 -11 años con edad auditiva no compensada

Author (s) / Autor (es):

Páez Pinilla, Prado Ortiz, Suárez Méndez, Carvajalino Monje, Grimaldos Franco, \& Rivera Quintero

Keywords / Palabras Clave:

[en]: Children, Postural balance, Vestibular diseases, Hearing loss, Cochlear implant, Hearing age, Occupational therapy.

[es]: Niños, Equilibrio postural, Enfermedades vestibulares, Pérdida auditiva, Implante coclear, Edad auditiva, Terapia ocupacional

Submited: 2021-04-02

Acepted: 2021-04-12

\section{Resumen}

La posturografía permite evaluar la integración de los tres sistemas sensoriales que intervienen en el control postural y del equilibrio: el vestibular, el visual y el somatosensorial (Ronda, 2002). La presente investigación describe y analiza los resultados de la posturografía en niños entre seis y once años con pérdida auditiva quienes no han compensado su edad auditiva con respecto a su edad cronológica. Fueron evaluados dos grupos:

el grupo control conformado por 32 niños con sensibilidad auditiva periférica normal y el grupo de estudio, conformado por 16 niños con pérdida

auditiva, quienes usan amplificación auditiva y asisten a la Fundación CINDA para terapia auditiva con el fin de cerrar el GAP existente entre su edad cronológica y su edad auditiva. En

la evaluación audiológica se aplicó anamnesis, revisión de historia clínica, audiometría tonal para la verificación de la sensibilidad auditiva periférica y posturografía, con un equipo de la marca NeuroCom, se realizaron las siguientes pruebas: Integración sensorial en balance-modificado (CTSIB), límites de estabilidad (LOS) y distribución de peso (WHT). En los resultados se encontraron diferencias estadísticamente significativas entre los dos grupos de estudio para las tres pruebas en distintas variables. Como conclusión se pudo determinar que existen diferencias estadísticamente significativas entre el grupo control y el grupo de estudio. La intervención con terapia ocupacional influenció positivamente los

resultados en la posturografía, sobre todo en el grupo de 10 a 11 años. Se observaron diferencias sucesivas en los tres grupos de edad, indicando un componente de neuromaduración.
Abstract

Posturography allows evaluating the integration of the three sensory systems involved in postural and balance control: vestibular, visual and somatosensory (Ronda, 2002). This research describes and analyzes the results of posturography in children between six and

eleven years old with hearing loss who have not compensated their hearing age with respect to their chronological age. Two groups were evaluated: the control group made up of 32 children with normal peripheral hearing sensitivity and the study group, made up of 16 children

with hearing loss, who use hearing amplification and attend the CINDA Foundation for hearing therapy in order to close the hearing loss. GAP

between your chronological age and your hearing age. In the audiological evaluation, anamnesis, review of clinical history, tonal audiometry were applied to verify peripheral hearing sensitivity and posturography, with a NeuroCom brand equipment, the following tests were performed: Modified-balance sensory integration (CTSIB), stability limits (LOS) and weight distribution

$(\mathrm{WHT})$. In the results, statistically significant differences were found between the two study groups for the three tests in different variables. In conclusion, it was possible to determine that there are statistically significant differences between the control group and the study group.

The occupational therapy intervention positively influenced the results in posturography, especially in the 10 to 11 -year-old group. Successive differences were observed in the three age groups, indicating a neuromaturation component.

\section{Citar como:}

Páez Pinilla, A. T., Prado Ortiz, K. D., Suárez Méndez, D. M., Carvajalino Monje, I. ., Grimaldos Franco, Y. ., \& Rivera Quintero, X. (2021). Posturografía En Niños Entre 6 -11 Años Con Edad Auditiva No Compensada. Revista Areté , 21 (1), 1-19. Obtenido de: arete.ibero.edu.co/article/ view/2124

Diana Marcela Suárez Méndez, Pre

Source | Filiacion:

Universidad Nacional de Colombia

BIO:

Fonoaudióloga, Universidad Nacional de Colombia (UNAL).

City I Ciudad:

Bogotá

Irma Carvajalino Monje, Pre

Source | Filiacion:

Universidad Nacional de Colombia

BIO:

Fonoaudióloga, Especialista en Audiología

Directora de Audiología, Centro para la

investigación e información para el deficiente

auditivo CINDA

City I Ciudad:

Bogotá
Yadira Grimaldos Franco, Presp

Source | Filiacion:

Universidad Nacional de Colombia BIO:

Fonoaudióloga, Especialista en Audiología, Audióloga consultora empresa BIOSIGMA de Colombia S.A.S.

City I Ciudad:

Bogotá

\section{Ximena Rivera Quintero, Pre Source I Filiacion \\ CINDA \\ BIO:}

Terapeuta Ocupacional, certificada en Integración Sensorial, CINDA.

City I Ciudad:

Bogotá

kdpradoo@unal.edu.co 


\title{
Posturografía en niños entre 6 -11 años con edad auditiva no compensada
}

Posturography in children between 6 -11 years old with non-compensated hearing age

\author{
Amanda Teresa Páez Pinilla \\ Karen Dinaluz Prado Ortiz \\ Diana Marcela Suárez Méndez \\ Irma Carvajalino Monje (Coautor) \\ Yadira Grimaldos Franco (Coautor) \\ Ximena Rivera Quintero (Coautor)
}

\section{Introducción}

Es importante que el abordaje para la evaluación, diagnóstico e intervención del niño con deficiencia auditiva se enfoque integralmente, es decir, se considere tanto al sistema auditivo como al vestibular, dadas las estrechas relaciones anatómicas, fisiológicas y patológicas existentes entre los dos. Se toma como sistema periférico al oído interno, que es el órgano sensorial para ambos sistemas, con la cóclea como el órgano sensorial auditivo, el utrículo, el sáculo y los tres canales semicirculares como los órganos sensoriales vestibulares. El VIII par craneal o el estato-acústico, con sus ramas, coclear, vestibular inferior y vestibular superior, es el conductor de los impulsos nerviosos hacia el sistema central. El tallo cerebral, el tálamo, la corteza cerebral y el cuerpo calloso son las estructuras integradoras centrales de la información proveniente desde las estructuras periféricas, responsables del procesamiento simétrico.

Así mismo, la complejidad del sistema auditivo y vestibular se explica por las múltiples vías, decusaciones, sinapsis y asociaciones con otros sistemas sensoriales, tanto a nivel periférico como central, de igual forma (Rodrigo., 2007) señala que: "Las ondas sonoras que viajan por el aire estimulan los receptores vestibulares del oído interno para mandar impulsos a los centros auditivos del tallo cerebral. Los núcleos centrales del VIII par procesan los impulsos auditivos junto con los impulsos provenientes del sistema vestibular" (p.11). Entonces, el sistema nervioso central debe integrar la información sensorial proveniente desde los sistemas auditivo, vestibular, visual y somatosensorial, para el desarrollo y control del equilibrio estático y dinámico. 
Por ello, para la valoración objetiva de la estabilidad postural en bipedestación existe la Posturografía Computarizada (Computarized Posturography, CP), técnica que se puede definir como el control del centro de masas con respecto a la base de soporte, en situaciones estáticas y dinámicas. Para esta, se emplea un equipo llamado posturógrafo, el cual cuenta con un software especializado para captar y procesar la información registrada a través de una plataforma dotada con sensores de presión de la planta de los piesy algunos traen también gafas de luz infrarroja, para registrar y medir el control postural y los movimientos oculares espontáneos, reflejos y voluntarios, durante los desplazamientos corporales y de la cabeza. Además, capta y mide los posibles nistagmos o actividad anormal de los ojos y el control inapropiado del cuerpo para el control del equilibrio (Civantos, 2018).

Para la intervención exitosa del deficiente auditivo, hace diferencia la detección, identificación e intervención temprana, con ayudas auditivas electroacústicas y técnicas de rehabilitación antes de cumplir con los periodos críticos de desarrollo neurológico, importantes por plasticidad en la especialización funcional cortical. Al respecto, cabe mencionar que la edad auditiva según (Cole, 2015) es un concepto útil porque da cuenta del tiempo que el niño necesita para reconocer y procesar la información auditiva e inicia cuando el niño lleva tecnología auditiva apropiadamente seleccionada y ajustada, es decir, que se asume desde el inicio del proceso de intervención, cuando el deficiente auditivo tiene acceso al sonido e inicia un proceso formal de desarrollo de sus habilidades auditivas. Específicamente, para el caso de niños con implantes cocleares, el inicio de la edad auditiva se toma como la fecha en la cual se enciende el implante coclear, después del proceso de cicatrización, estimado en la mayoría de los casos, como un mes después de la cirugía de implantación.

Con respecto a lo anterior, llama la atención que en algunos niños este proceso se produce con mayor lentitud y el cierre de la diferencia o GAP entre edad auditiva y edad cronológica, no se alcanza en el tiempo esperado. Por esto, se buscan entonces otros factores asociados que puedan estar limitando el desarrollo normal de este proceso y uno de ellos podría ser la diferencia en el desarrollo paralelo del sistema vestibular, pues cuando se detecta la deficiencia auditiva en los niños, la intervención se enfoca en el desarrollo de las habilidades auditivas y del lenguaje oral, y se desconoce la importancia del desarrollo paralelo del sistema vestibular y del equilibrio.

Por los anteriores planteamientos, en la presente investigación se describen y analizan los resultados de la posturografía estática en niños con deficiencia auditiva, quienes no han logrado cerrar el GAP o diferencia entre la edad auditiva y la edad cronológica. Se espera que los resultados se puedan utilizar para proponer actividades de intervención que integren el desarrollo de los sistemas auditivo, vestibular, visual y somatosensorial.

\section{Metodología}

Estudio dentro del paradigma cuantitativo, con método descriptivo, correlacional y técnica aplicada, para establecer las posibles relaciones entre las variables estudiadas; para la resolución de los problemas estadísticos generados en el presente estudio se realizaron los siguientes análisis:

Análisis descriptivo de los datos.

Intervalos de confianza para la propuesta de una normativa en niños, para próximos diagnósticos.
Análisis de Varianza para ver los efectos de cambio en las diferentes situaciones durante la prueba del CTSIB.

Comparación de grupos por medio de pruebas no paramétricas, tales como la prueba de Wilcoxon.

La población la conformaron niños entre 6 y 11 años, con pérdida auditiva neurosensorial bilateral, quienes asistían regularmente a la fundación CINDA para intervención integral (fonoaudiológica, audiológica, psicológica, pedagógica y de terapia ocupacional). Los sujetos fueron identificados por censo entre quienes cumplieron los criterios de inclusión y exclusión. El censo también fue depurado por quienes no contaban con consentimiento informado de sus padres o acudientes, para dar cumplimiento con las normas éticas relacionadas con la atención en salud de menores de edad. Para el grupo de control, participaron 32 niños con sensibilidad auditiva periférica dentro de límites normales, en el mismo rango de edades, sin antecedentes neuro-otológicos conocidos.

\section{Resultados}

En primer lugar, se tuvo un grupo de control conformado por 32 niños en el rango de edades entre 6 y 11 años, los cuales presentaban sensibilidad auditiva periférica bilateral dentro de parámetros normales, verificada previamente por la realización de la anamnesis, audiometría tonal y otoscopia, antes de llevar a cabo la posturografía.

Posteriormente, se obtiene una muestra de niños igualmente entre 6 y 11 años, con deficiencia auditiva neurosensorial, quienes asistían regularmente a la fundación Centro de Investigación e Información en Deficiencias Auditivas (CINDA), con los cuales se aplicaron ciertos criterios de inclusión o exclusión dentro de la muestra. El grupo de niños con pérdida auditiva incluidos en la muestra de estudio está conformado por 16 niños en total. A este grupo de niños se les realizó la anamnesis, la revisión de la historia clínica en donde se encuentra reportada la audiometría más reciente, los resultados de las intervenciones fonoaudiológicas y de terapia ocupacional, previo a la realización de la posturografía.

\section{Perfil demográfico}

\section{Grupo control.}

Para el análisis demográfico del grupo control, se obtuvieron los siguientes resultados: el grupo control estuvo conformado por 17 niños y 15 niñas (Gráfico 1). Además, en el gráfico 2 se reporta la distribución del grupo por rango de edades, expresado en 12 niños/as entre seis y siete años, 13 niños/as entre ocho y nueve años y, por último, siete niños/as entre 10 y 11 años, para un total de 32 sujetos. Se presenta en el gráfico 3, el grado de escolaridad alcanzado por los niños del grupo control, en donde 11 sujetos estaban cursando el grado primero de primaria, tres el grado segundo, ocho habían alcanzado el grado de escolaridad tercero, seis el grado de escolaridad cuarto, tres el grado de escolaridad quinto, y sólo uno estaba cursando sexto de bachillerato 


\section{Gráfico de barras por sexo}

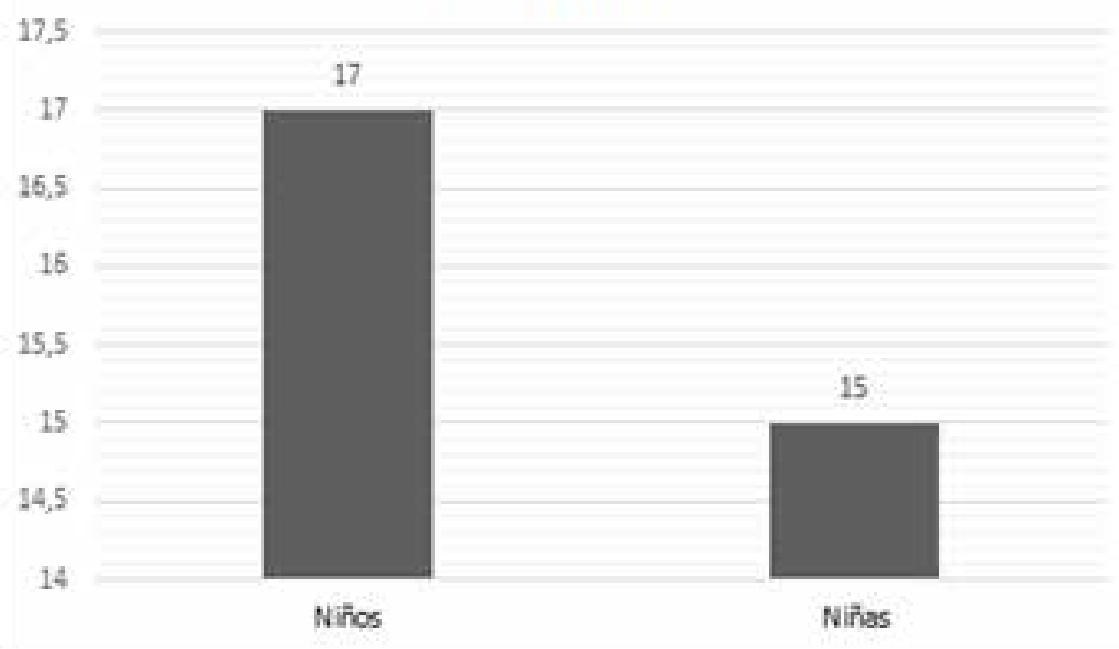

Gráfico 1. Gráfico de barras por sexo. Elaboración propia.

\section{Rangos de edad}

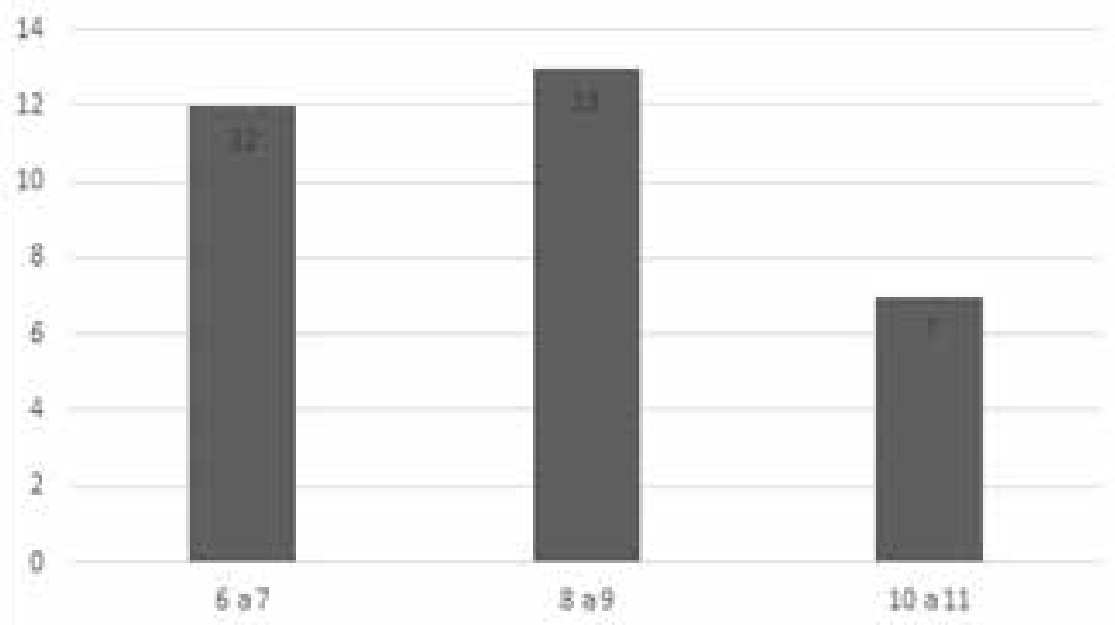

Gráfico 2. Gráfico de barras por rangos de edad. Elaboración propia.

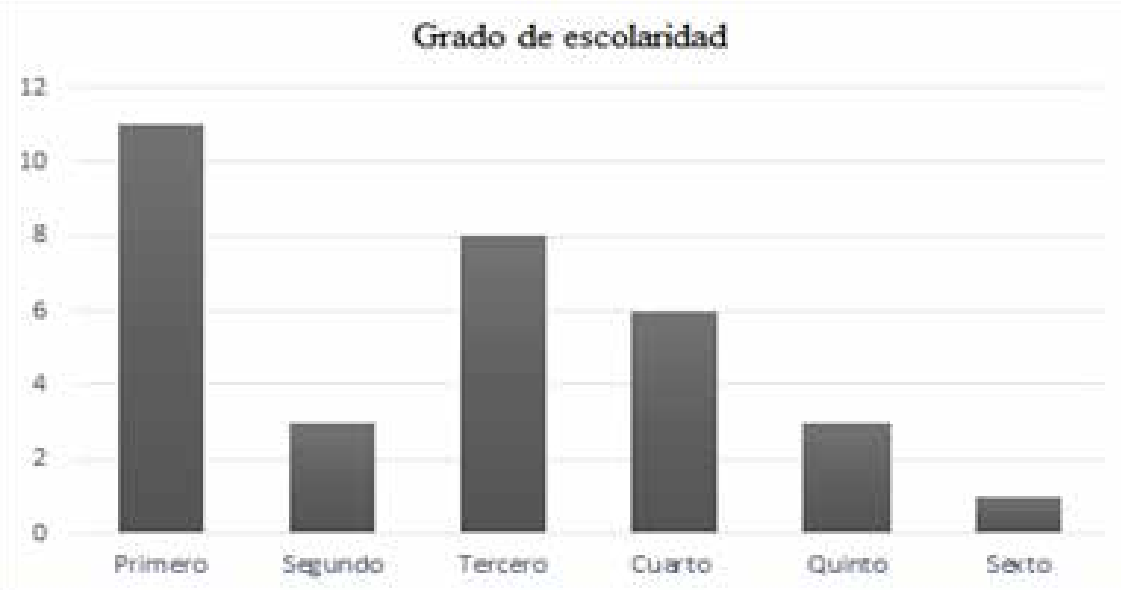

Gráfico 3. Gráfico de barras por grado de escolaridad. Elaboración propia.

\section{Grupo de estudio.}

Del grupo en estudio (con pérdida auditiva) se obtienen los siguientes resultados: nueve sujetos eran niños y siete eran niñas (gráfico 4). Se puede observar en el gráfico 5 correspondiente a rangos por edad, que, del total de los 16 sujetos, cuatro niños/as pertenecen al grupo de seis y siete años; seis niños/as pertenecen a las edades entre ocho y nueve años, $y$, finalmente, seis pertenecen a niños/as entre las edades de 10 y 11 años. Por último, para la caracterización del grado de escolaridad se presenta el gráfico 6, donde tres niños cursaban el grado preescolar, dos niños habían alcanzado el grado de escolaridad primero de primaria, tres el grado de escolaridad segundo, cuatro el grado de escolaridad tercero, tres el grado de escolaridad cuarto, y finalmente, un sujeto había alcanzado el grado de escolaridad quinto de primaria. Es importante destacar que los niños pertenecientes al grupo de estudio asistían a colegios regulares con normo-oyentes en donde recibían su formación académica, y el apoyo pedagógico y la rehabilitación la recibían en la jornada contraria en CINDA.

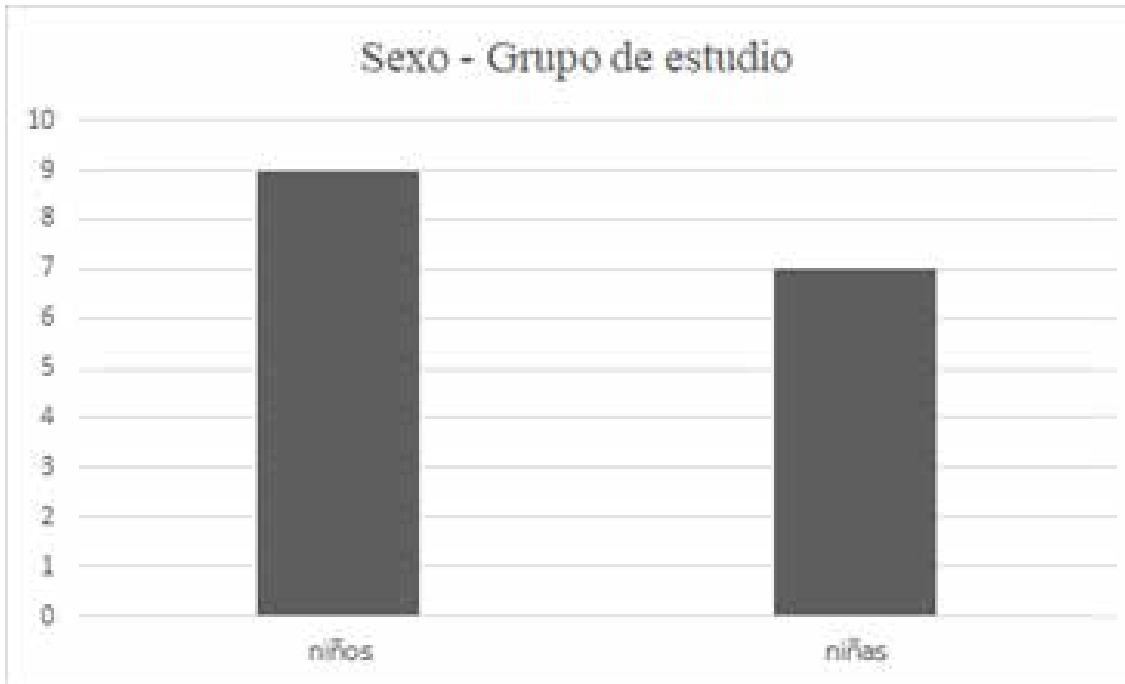

Gráfico 4. Gráfico de barras por sexo del grupo de estudio. Elaboración propia

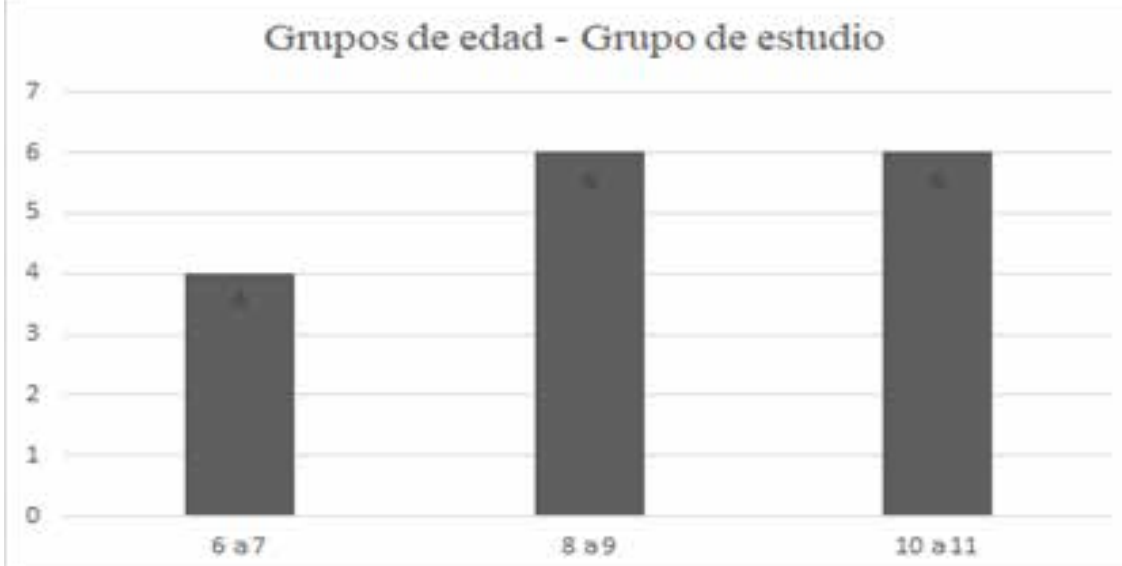

Gráfico 5. Gráfico de barras por rangos de edad del grupo de estudio. Elaboración propia

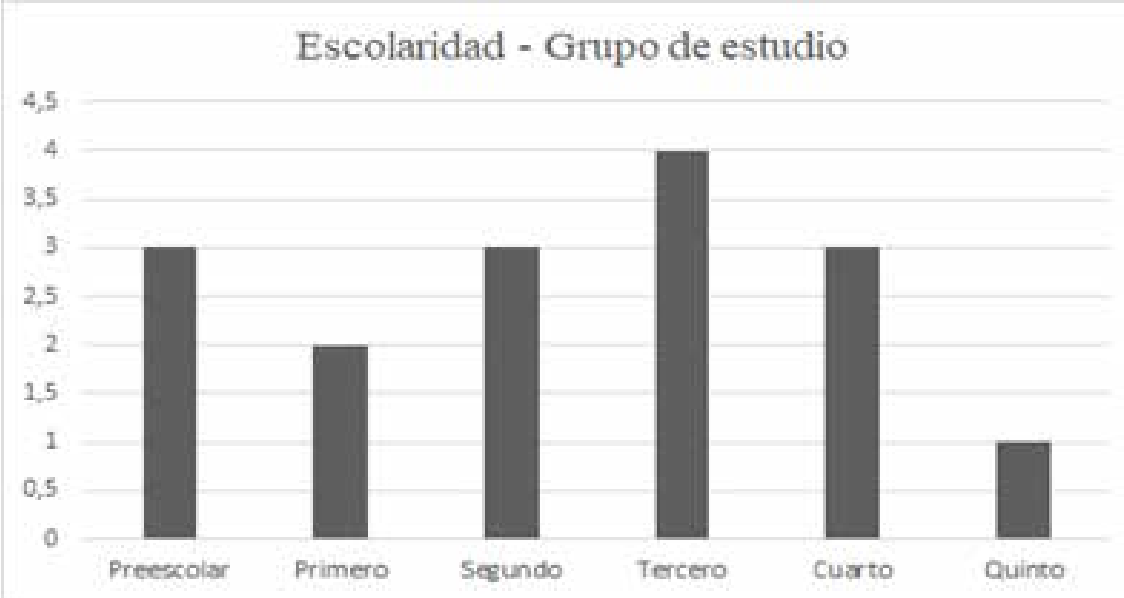

Gráfico 6. Gráfico de barras por escolaridad alcanzada del grupo de estudio. Elaboración propia.

Se analizaron los antecedentes pre, peri y postnatales de los 16 sujetos en estudio, de estos, siete reportaron complicaciones prenatales, durante la gestación; 12 niños presentaron dificultades durante el parto o antecedentes perinatales y en diez niños, los padres reportaron complicaciones en los primeros 28 días después del parto (Tabla 1). Continuando con antecedentes del desarrollo, todos los niños por tener pérdida auditiva presentaban dificultad para la adquisición y/o el desarrollo del lenguaje. Los padres reportaron que, cinco niños tenían dificultad para el desarrollo motor, seis dificultades en el equilibrio y seis con dificultades del aprendizaje. 
Posturografía en niños entre 6 -11 años con edad auditiva no compensada

Tabla 1. Antecedentes reportados para el grupo control

\begin{tabular}{|c|c|c|}
\hline \multicolumn{3}{|c|}{ Antecedentes } \\
\hline & Reporta & No reporta \\
\hline Prenatales & 7 & 9 \\
\hline Perinatales & 12 & 4 \\
\hline Postnatales & 10 & 6 \\
\hline
\end{tabular}

Se indaga sobre antecedentes médicos y ambientales: en seis niños la pérdida auditiva se asoció a kernícterus, bronquiolitis, amigdalitis o nódulos vocales. Siete recibieron algún procedimiento quirúrgico, uno reportó tratamiento farmacológico, tres presentaron trauma de cabeza o cuello y dos se encontraban expuestos a ruido, polución o vibración (Tabla 2).

Tabla 2 Antecedentes del desarrollo, médicos y ambientales del grupo control

\begin{tabular}{|c|c|c|}
\hline Variable & Normal & Dificultad \\
\hline Desarrollo Motor & 11 & 5 \\
\hline Equilibrio & 10 & 6 \\
\hline Aprendizaje & 10 & 6 \\
\hline Patológicos & 10 & 6 \\
\hline Quirúrgicos & 9 & 7 \\
\hline Medicamentosos & 15 & 1 \\
\hline Traumatológicos & 13 & 3 \\
\hline Ambientales & 14 & 2 \\
\hline
\end{tabular}

\section{Perfil audiológico}

\section{Grupo control.}

Previo a la realización de la posturografía de cada niño del grupo control, se realizó una otoscopia y una audiometría tonal, encontrándose que todos obtuvieron un diagnóstico audiológico de sensibilidad auditiva periférica bilateral dentro de parámetros normales.

\section{Grupo de estudio.}

A continuación, se reportan las características de la pérdida auditiva de los sujetos de estudio, distribuidos en configuración y grado de pérdida auditiva. Es pertinente aclarar que todos los niños tenían pérdida auditiva de tipo neurosensorial y no se encontraron componentes mixtos. En relación con la configuración de la pérdida auditiva, expuesto en la tabla 3, tenemos que seis niños presentaban pérdida auditiva asimétrica y diez presentaban pérdida auditiva simétrica. Enseguida, en la tabla 4, se encuentran los grados de pérdida auditiva, distribuidos así: dos niños/as presentaban pérdida auditiva de grado moderado, tres tenían pérdida auditiva de grado severo y 11 pérdida auditiva de grado profundo.
Tabla 3. Configuración audiológica del grupo de estudio

\begin{tabular}{|c|c|c|c|}
\hline \multicolumn{4}{|c|}{ Configuración de pérdida auditiva } \\
\hline Rango de Edad & Asimétrico & Simétrico & Total general \\
\hline 6 a 7 & 2 & 2 & 4 \\
\hline 8 a 9 & 2 & 4 & 6 \\
\hline 10 a 11 & 2 & 4 & 6 \\
\hline Total general & 6 & 10 & 16 \\
\hline
\end{tabular}

Tabla 4. Grado de pérdida auditiva del grupo de estudio.

\begin{tabular}{|c|c|c|c|c|}
\hline \multicolumn{5}{|c|}{ Grado de pérdida auditiva } \\
\hline $\begin{array}{c}\text { Rango de } \\
\text { Edad }\end{array}$ & Moderado & Severo & Profundo & $\begin{array}{c}\text { Total } \\
\text { general }\end{array}$ \\
\hline 6 a 7 & 0 & 1 & 3 & 4 \\
\hline 8 a 9 & 1 & 1 & 4 & 6 \\
\hline 10 a 11 & 1 & 1 & 4 & 6 \\
\hline $\begin{array}{c}\text { Total } \\
\text { general }\end{array}$ & 2 & 3 & 11 & 16 \\
\hline
\end{tabular}

Las ayudas auditivas utilizadas por los niños, se presentan en la tabla 5. En el grupo de seis a siete años, dos niños usaban amplificación en ambos oídos, uno con implante coclear bilateral y otro con audífono bilateral. Un usuario presentaba únicamente implante coclear en el oído derecho y el niño restante no usaba amplificación. Para el siguiente grupo de ocho a nueve años, cuatro niños usaban amplificación en ambos oídos, de los cuales dos sujetos tenían implante coclear bilateral y dos contaban con audífonos en ambos oídos; un infante usaba implante coclear únicamente en oído izquierdo y otro solamente implante coclear en oído derecho. Finalizando con el grupo de 10 a 11 años, todos los seis niños presentaban amplificación bilateral, reportándose dos con implante coclear en ambos oídos, dos con audífonos bilateralmente y dos con una amplificación bimodal, en donde presentaban implante coclear en oído derecho y audífono en oído izquierdo.

Tabla 5. Ayudas auditivas usadas por el grupo de estudio

\begin{tabular}{|c|c|c|c|c|c|}
\hline $\begin{array}{c}\text { Rango de } \\
\text { edad }\end{array}$ & $\begin{array}{l}\text { Ambos } \\
\text { oídos }\end{array}$ & $\begin{array}{c}\text { No tiene } \\
\text { amplificación }\end{array}$ & OI & OD & $\begin{array}{l}\text { Total } \\
\text { general }\end{array}$ \\
\hline 6 a 7 & $\begin{array}{l}1 \mathrm{IC} \\
1 \mathrm{Au}\end{array}$ & 1 & 0 & $1 \mathrm{IC}$ & 4 \\
\hline 8 a 9 & $\begin{array}{l}2 \mathrm{IC} \\
2 \mathrm{Au}\end{array}$ & 0 & $1 \mathrm{IC}$ & $1 \mathrm{IC}$ & 6 \\
\hline 11 & $\begin{array}{c}2 \mathrm{IC} \\
2 \mathrm{Au} \\
2 \mathrm{IC} \text { en OD + } \\
\text { Au en OI }\end{array}$ & 0 & 0 & 0 & 6 \\
\hline Total & 12 & 1 & 1 & 2 & 16 \\
\hline
\end{tabular}

\section{Perfil comunicativo}

\section{Grupo control.}

El grupo control presentaba habilidades auditivas y comunicativas conservadas y acordes a su edad, por lo que no recibían ningún tipo de apoyo o rehabilitación terapéutica, por lo tanto, se asume que su edad auditiva corresponde a la edad cronológica. 


\section{Grupo de estudio.}

En las habilidades comunicativas se incluyeron las variables de intervención auditiva, el GAP, la edad auditiva y el diagnóstico comunicativo identificado en la fundación CINDA. En cuanto a la intervención auditiva que reciben los usuarios, 14 niñosseencuentraban en habilitación auditiva con Terapia Auditiva Verbal (TAV) y dos niños recibían terapia McGinnis, como se presenta en el gráfico 7.

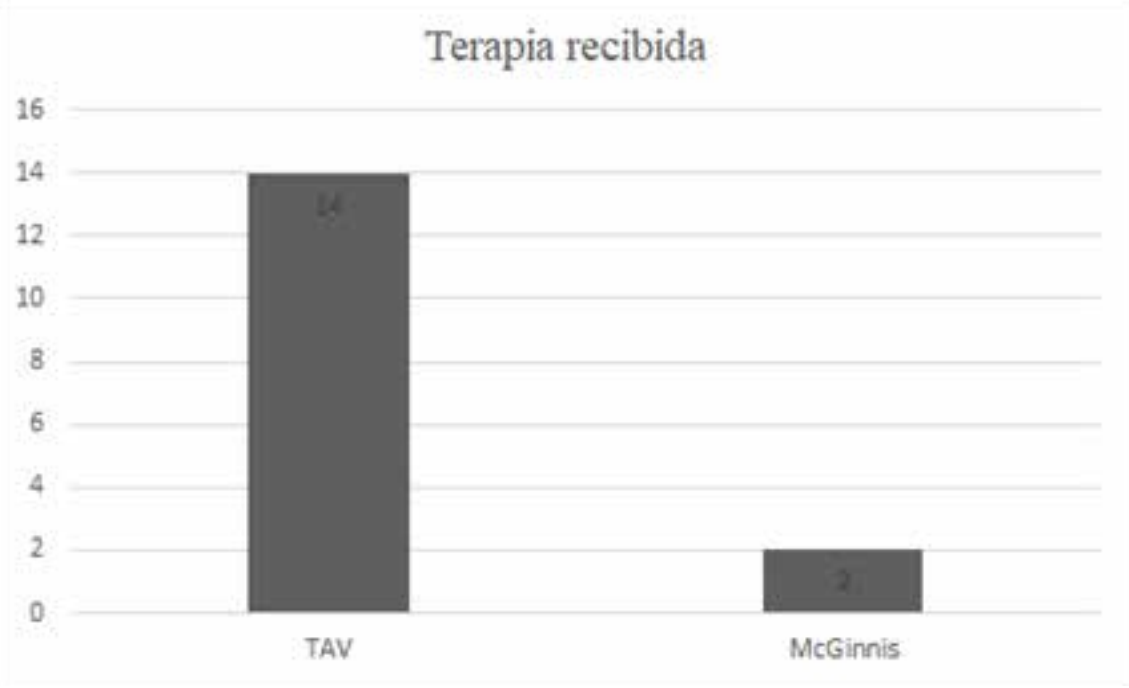

Gráfico 7. Gráfico de barras por terapia recibida por el grupo de estudio. Elaboración propia

Pasando a la variable de GAP, como se observa en la tabla 6, el valor mínimo y máximo existentes son 3 y 100 meses respectivamente, lo que ubica la media en 36,38 meses; respecto a la edad auditiva, que se presenta en la misma tabla, donde valor mínimo correspondía a 13 meses y el máximo a 117 meses, con una media de 71,75 meses.

Tabla 6. Valores del GAP y la edad auditiva, expresados en meses, del grupo de estudio

\begin{tabular}{|c|c|c|}
\hline Valores & GAP & Edad Auditiva \\
\hline Valor mínimo & 3 & 13 \\
\hline Primer cuartil & 21,5 & 51,25 \\
\hline Mediana & 35,5 & 72,5 \\
\hline Media & 36,38 & 71,75 \\
\hline Tercer cuartil & 46,25 & 97,75 \\
\hline Valor máximo & 100 & 117 \\
\hline
\end{tabular}

En cuanto al diagnóstico comunicativo, todos los pacientes presentaban código actualizado al CIE-10 F.808 que corresponde a Otros Trastornos del Desarrollo del Habla y del Lenguaje.

\section{Posturografía}

\section{Grupo control.}

Con los datos obtenidos de los 32 niños que conforman el grupo control, se elaboró la normativa del posturógrafo para dicha población, subdividida por rangos de edad, de seis a siete años, ocho a nueve y diez a once años. Los resultados se resumen en las siguientes tablas:
Tabla 7. Valores de normativa de la prueba de Integración sensorial del Balance-Modificado (CTSIB), en los tres grupos de edad, de los sujetos con desarrollo normal y sin antecedentes neuro-otológicos.

\begin{tabular}{|c|c|c|c|c|c|}
\hline Variable & $6-7$ & $8-9$ & $10-11$ & $\begin{array}{c}\text { Unidades } \\
\text { de } \\
\text { medida }\end{array}$ & $\begin{array}{c}\text { Normativa } \\
\text { adulta (20- } \\
39)\end{array}$ \\
\hline Firm-OA & $0.56-0.80$ & $0.52-0.69$ & $0.40-0.84$ & Grado/seg & $0-0.35$ \\
\hline Firm-OC & $0.73-1.14$ & $0.56-0.74$ & $0.49-0.90$ & Grado/seg & $0-0.45$ \\
\hline Foam-OA & $0.99-1.23$ & $0.84-1.09$ & $0.91-1.40$ & Grado/seg & $0-0.75$ \\
\hline Foam-OC & $1.73-2.51$ & $1.39-2.12$ & $1.61-2.77$ & Grado/seg & $0-1.9$ \\
\hline Comp & $1.06-1.41$ & $0.85-1.13$ & $0.89-1.47$ & Grado/seg & $0-0.8$ \\
\hline Alin COG & $24-46$ & $21-27$ & $21-38$ & Porcentaje & \\
\hline
\end{tabular}

Firm-OA: Superficie firme, ojos abiertos. Firm-oc: Superficie firme, ojos cerrados. Foam-OA: Sup erficie inestable, ojos abiertos. Foam-OC: Superficie inestable, ojos cerrados. Comp: compilado o composite. Alin COG: Alineamiento del centro de gravedad.

Los valores obtenidos reflejan que, a medida que aumenta la dificultad de la prueba, al quitar el apoyo visual y/o somatosensorial, los valores de la velocidad de oscilación aumentan de manera directamente proporcional, por ello, el menor valor resultante es el de la primera condición, en donde se tiene superficie firme y ojos abiertos. La condición en la que se obtuvo el mayor valor corresponde a la superficie inestable y ojos cerrados. Este patrón de presentación se mantiene para los tres grupos de edad, reflejado igualmente en la normativa para adultos.

En los resultados anteriores se encuentra que, entre los dos primeros grupos de edad sí hay disminución en los rangos de los valores. Dicha disminución se atribuye a la neuromaduración y las habilidades adquiridas. En el tercer grupo no se aprecia esta diferencia, lo cual se puede atribuir a que el grupo control para dicha edad fue más pequeño, por ello se sugiere para próximos estudios similares ampliar el número de la población, pues el presente trabajo es un estudio exploratorio.

Se observa también que la Alineación del Centro de Gravedad (COG) en el grupo control, el valor máximo correspondió al grupo de seis y siete años y el menor valor, a los niños de ocho, nueve, diez y once años, lo que se atañe al hecho de que los niños más grandes, gracias a la neuromaduración, poseen una lateropulsión menos evidente y menor riesgo de caída de altura corporal.

Comparando la normativa de los niños en los tres grupos de edad con la normativa de adultos para la edad de 20 a 39 años, se encuentra que, los rangos de la normalidad son más amplios para los niños, e inician en valores mayores que el de los adultos, lo que está en concordancia con la neuromaduración. 


\section{Posturografía en niños entre 6 -11 años con edad auditiva no compensada}

Tabla 8. Valores de normativa de la prueba de Distribución de Peso (WHT), en los tres grupos de edad, de los sujetos con desarrollo normal, sin antecedentes neuro-otológicos.

\begin{tabular}{|c|c|c|c|c|c|}
\hline Variable & $6-7$ & $8-9$ & $10-11$ & $\begin{array}{c}\text { Unidades } \\
\text { de medida }\end{array}$ & $\begin{array}{c}\text { Normativa } \\
\text { adulta (20-39) }\end{array}$ \\
\hline 0-Izq & $49.58-52.67$ & $47.77-0.92$ & $44.81-51.00$ & Grados & $40-60$ \\
\hline 0-Dere & $47.00-50.25$ & $48.85-52.15$ & $48.71-54.99$ & Grados & $40-60$ \\
\hline 30-Izq & $49.51-52.75$ & $46.54-51.62$ & $43.00-49.57$ & Grados & $40-60$ \\
\hline 30-Dere & $48.17-51.50$ & $47.46-52.69$ & $50.29-55.79$ & Grados & $40-60$ \\
\hline 60-Izq & $48.42-53.25$ & $47.62-51.69$ & $43.09-50.57$ & Grados & $40-60$ \\
\hline 60-Dere & $46.17-51.50$ & $48.26-52.46$ & $49.14-56.86$ & Grados & $40-60$ \\
\hline 90-Izq & $46.00-53.17$ & $46.88-52.31$ & $46.48-53.43$ & Grados & $40-60$ \\
\hline 90-Dere & $46.77-53.58$ & $47.73-52.85$ & $46.43-52.86$ & Grados & $40-60$ \\
\hline
\end{tabular}

Esta prueba arrojó datos variables entre las edades, sin seguir un patrón específico, a excepción de los más pequeños (seis a siete años), quienes mostraron que la distribución equitativa del peso se va perdiendo a medida que aumenta el ángulo de flexión de las rodillas.
Así mismo, los valores de esta prueba no presentaron diferencias entre niños y adultos, pues en estos últimos se maneja un rango de normalidad bastante amplio, por ello, la presente prueba no estaría ofreciendo ninguna diferencia estadística

Tabla 9. Valores de normativa para la prueba de Límites de Estabilidad (LOS), en los tres grupos de edad, de los sujetos con desarrollo normal, sin antecedentes neuro-otológicos.

\begin{tabular}{|c|c|c|c|c|c|}
\hline Variable & $6-7$ & $8-9$ & $10-11$ & Unidad de medida & $\begin{array}{c}\text { Normativa adulto } \\
(20-39)\end{array}$ \\
\hline F-RT & $0.45-1.16$ & $0.71-1.44$ & $0.65-1.05$ & Seg & $0-0.9$ \\
\hline F-MVL & $3.26-5.05$ & $4.39-7.83$ & $5.07-10.74$ & Grado/seg & $2.9-10$ \\
\hline F-EPE & $46-82$ & $51-76$ & $52-92$ & Porcentaje & $78-120$ \\
\hline F-MXE & $86-102$ & $81-100$ & 73-109 & Porcentaje & $90-120$ \\
\hline F-DCL & $76-86$ & $59-83$ & $49-84$ & Porcentaje & $69-100$ \\
\hline RF-RT & $0.73-1.12$ & $0.83-1.24$ & $0.56-1.13$ & Seg & \\
\hline RF-MVL & $4.57-7.36$ & $5.01-7.62$ & $4.11-5.42$ & Grado/seg & \\
\hline RF-EPE & $58-89$ & $57-76$ & $49-90$ & Porcentaje & \\
\hline RF-MXE & $87-107$ & $87-101$ & $73-102$ & Porcentaje & \\
\hline RF-DCL & $59-74$ & $59-79$ & $32-77$ & Porcentaje & \\
\hline R-RT & $0.71-1.07$ & $0.64-1.03$ & $0.67-1.11$ & Seg & $0-0.9$ \\
\hline R-MVL & $5.61-8.22$ & $4.46-7.46$ & $5.03-6.84$ & Grado/seg & $2.9-10$ \\
\hline R-EPE & $69-85$ & $59-77$ & $50-86$ & Porcentaje & $78-120$ \\
\hline R-MXE & $80-94$ & $75-89$ & $76-94$ & Porcentaje & $90-120$ \\
\hline $\mathrm{R}-\mathrm{DCL}$ & $70-80$ & $68-80$ & $63-77$ & Porcentaje & $68-100$ \\
\hline RB-RT & $0.58-1.08$ & $0.64-1.03$ & $0.40-0.93$ & Seg & \\
\hline RB-MVL & $3.89-5.99$ & $3.99-5.76$ & $3.45-6.52$ & Grado/seg & \\
\hline RB-EPE & $51-74$ & $55-79$ & $50-72$ & Porcentaje & \\
\hline RB-MXE & $66-90$ & $74-92$ & $86-103$ & Porcentaje & \\
\hline RB-DCL & $29-59$ & $42-65$ & $48-71$ & Porcentaje & \\
\hline B-RT & $0.33-0.64$ & $0.50-0.81$ & $0.28-0.81$ & Seg & $0-0.9$ \\
\hline
\end{tabular}




\begin{tabular}{|c|c|c|c|c|c|}
\hline Variable & $6-7$ & $8-9$ & $10-11$ & Unidad de medida & $\begin{array}{c}\text { Normativa adulto } \\
(20-39)\end{array}$ \\
\hline B-MVL & $2.85-5.24$ & $2.70-4.04$ & $3.21-5.76$ & Grado/seg & $0.9-10$ \\
\hline B-EPE & $55-80$ & $47-65$ & $4-76$ & Porcentaje & $19-120$ \\
\hline B-MXE & $61-86$ & $70-92$ & $75-92$ & Porcentaje & $29-120$ \\
\hline$B-D C L$ & $33-58$ & $43-71$ & $46-67$ & Porcentaje & $19-100$ \\
\hline LB-RT & $0.53-0.98$ & $0.70-1.14$ & $0.61-0.90$ & Seg & \\
\hline LB-MVL & $5.52-7.55$ & $3.80-6.19$ & $4.61-6.29$ & Grado/seg & \\
\hline LB-EPE & $59-80$ & $47-71$ & $47-77$ & Porcentaje & \\
\hline LB-MXE & $75-92$ & $80-100$ & $75-90$ & Porcentaje & \\
\hline LB-DCL & $34-59$ & $53-72$ & $28-68$ & Porcentaje & \\
\hline L-RT & $0.86-1.06$ & $0.75-1.16$ & $0.43-0.78$ & Seg & $0-0.9$ \\
\hline L-MVL & $5.39-7.89$ & $4.50-6.26$ & $4.43-7.63$ & Grado/seg & $2.9-10$ \\
\hline L-EPE & $64-79$ & $54-76$ & $47-79$ & Porcentaje & $78-120$ \\
\hline L-MXE & $79-93$ & $73-91$ & $69-89$ & Porcentaje & $90-120$ \\
\hline L-DCL & $73-82$ & $66-78$ & $43-84$ & Porcentaje & $68-100$ \\
\hline LF-RT & $0.69-0.85$ & $0.66-0.95$ & $0.60-1.07$ & Seg & \\
\hline LF-MVL & $5.6-7.99$ & $4.71-7.08$ & $4.32-7.20$ & Grado/seg & \\
\hline LF-EPE & $73-90$ & $60-80$ & $46-91$ & Porcentaje & \\
\hline LF-MXE & $90-107$ & $76-96$ & $70-104$ & Porcentaje & \\
\hline LF-DCL & $70-79$ & $43-78$ & $30-79$ & Porcentaje & \\
\hline Comp-RT & $0.70-0.86$ & $0.78-0.98$ & $0.65-0.78$ & Seg & $0-0.9$ \\
\hline Comp-MVL & $4.92-5.82$ & $4.29-5.56$ & $5.46-25.88$ & Grado/seg & $3-10$ \\
\hline Comp-EPE & $62-74$ & $57-68$ & $55-75$ & Porcentaje & $68-120$ \\
\hline Comp-MXE & $79-90$ & $77-90$ & $77-90$ & Porcentaje & $85-120$ \\
\hline Comp-DCL & $61-68$ & $62-74$ & $56-71$ & Porcentaje & 59-100 \\
\hline
\end{tabular}

Tiempo de reacción: adelante (F-RT), adelante a la derecha (RF-RT), derecha $(R-R T)$, atrás a la derecha (RB-RT), atrás (B-RT) atrás a la izquierda (LB-RT), izquierda (L-RT), adelante a la izquierda (LF-RT) y compilado (Comp-RT). Velocidad de movimiento: adelante (F-MVL), adelante a la derecha (RF-MVL), derecha (R-MVL), atrás a la derecha (RB-MVL), atrás (B-MVL) atrás a la izquierda (LB-MVL), izquierda (L-MVL), adelante a la izquierda (LF-MVL) y compilado (Comp-MVL). Punto de excursión

mínimo (EPE) y máximo (MXE): adelante (F-EPE, F-MXE), adelante a la derecha (RF-EPE, RF-MXE), derecha (R-EPE, R-MXE), atrás a la derecha (RB-EPE, RB-MXE), atrás (B-EPE, B-MXE) atrás a la izquierda (LB-EPE, LB-MXE), izquierda (L-EPE, L-MXE), adelante a la izquierda (LF-EPE, LF-MXE) y compilado (Comp-EPE, Comp-MXE).

Control direccional (DCL): adelante (F-DCL), adelante a la derecha (RF-DCL), derecha (R-DCL), atrás a la derecha (RB-DCL), atrás (B-DCL) atrás a la izquierda (LB$D C L)$, izquierda (L-DCL), adelante a la izquierda (LF-DCL) y compilado (Comp-DCL).

En cuanto a la prueba de Límites de Estabilidad (LOS), con base en los compilados, se observa que el tiempo de reacción en el grupo de niños entre 10 y 11 años es menor con respecto a los demás grupos, además, su velocidad de movimiento es mayor, por lo tanto, se movían más rápido y tardaban menos tiempo en reaccionar para iniciar el desplazamiento, atribuible a un mayor control postural generado por la neuromaduración subyacente. Los valores de punto de excursión máximo y mínimo (MXE y EPE, respectivamente) se muestran aumentados en los niños entre seis y siete años, mostrando que este grupo, estadísticamente hablando, eran quienes recorrían la mayor distancia dentro de los límites de estabilidad, lo que puede indicar que ellos eran quienes tenían un área mayor de estabilidad o que su relativo menor control postural les dificultaba regular su movimiento de desplazamiento. Por último, el grupo de ocho a nueve años presentó un mejor control direccional que sus pares, pues el desplazamiento producido está mejor orientado hacia el objetivo, indicando que no siempre una mayor rapidez de movimiento implica un mejor control del equilibrio. 


\section{Posturografía en niños entre 6 -11 años con edad auditiva no compensada}

\section{Grupo de estudio}

Con el fin de describir y analizar los resultados de la posturografía en el grupo de los niños en estudio, se realizaron las siguientes tablas:

Tabla 10. Valores de la prueba de Integración Sensorial del Balance - Modificado (CTSIB), en los tres grupos de edad, de los sujetos con deficiencia auditiva y edad auditiva no compensada.

\begin{tabular}{|c|c|c|c|c|c|}
\hline Prueba & Variable & 6 a 7 & 8 a 9 & 10 a 11 & $\begin{array}{c}\text { Unidades de } \\
\text { medida }\end{array}$ \\
\hline & Firm-OA & 0.8 & 0.85 & 0.6 & Grado/seg \\
\hline & Firm-OC & 0.9 & 0.8 & 0.6 & Grado/seg \\
\hline CTSIB & Foam-OA & 1.35 & 1.2 & 0.95 & Grado/seg \\
\hline & Comp & 1.3 & 1.25 & 0.9 & Grado/seg \\
\hline & Alin COG & 21 & 28 & 20 & Porcentaje \\
\hline
\end{tabular}

Firm-OA: Superficie firme, ojos abiertos. Firm-OC: Superficie firme, ojos cerrados. Foam-OA: Superficie inestable, ojos abiertos. Foam-OC: Superficie inestable, ojos cerrados. Comp: compilado o composite. Alin COG: Alineamiento del centro de gravedad.

Los resultados de la prueba de Integración Sensorial del BalanceModificado (CTSIB) en los sujetos en estudio, sugieren que a medida que se retiran los apoyos, ya sea visual y/o somatosensorial, los valores de oscilación van aumentando, siendo el menorvalor para la condición de plataforma firme y el mayor valor para la condición de plataforma inestable y ojos cerrados, este patrón concuerda con el presentado en la normativa; sin embargo, se observan valores de medianas más grandes en comparación con los niños del grupo control.

Debido a la distribución más equilibrada de la población por grupos de edad, en los sujetos en estudio sí se observa claramente cómo los resultados arrojados muestran la neuromaduración, disminuyendo la velocidad de oscilación a través de la edad.

La Alineación del Centro de Gravedad (COG) es una medida que permite vislumbrar el balanceo al inicio de cada desplazamiento en el LOS, donde $0 \%$ es el mínimo balanceo y $100 \%$ es el peor equilibrio. El grupo de ocho a nueve años se muestra por encima de la normativa, mientras que los dos grupos restantes se encuentran por debajo de ella. Esto indica que el grupo de ocho a nueve presentaban una mayor lateropulsión (28\%), lo que implica, de igual forma, un mayor riesgo de caída de altura corporal.

Tabla 11.Valores de la prueba de Distribución de Peso (WHT), en los tres grupos de edad, de los sujetos del grupo de estudio.

\begin{tabular}{|c|c|c|c|c|c|}
\hline Prueba & Variable & 6 a 7 & 8 a 9 & 10 a 11 & $\begin{array}{c}\text { Unidades de } \\
\text { medida }\end{array}$ \\
\hline & 0 -Izq & 50.5 & 48.5 & 50.5 & Porcentaje \\
\hline & 0 -Dere & 49.5 & 51.5 & 49.5 & Porcentaje \\
\hline & 30 -Izq & 45.5 & 47.5 & 49.5 & Porcentaje \\
\hline WHT & 30 -Dere & 54.5 & 52.5 & 50.5 & Porcentaje \\
\hline & 60 -Izq & 48.5 & 47.5 & 49.5 & Porcentaje \\
\hline & 90 -Izq & 50 & 47 & 51.5 & Porcentaje \\
\hline & 90 -Dere & 50 & 53 & 48.5 & Porcentaje \\
\hline
\end{tabular}

En la prueba de Distribución de Peso (WHT), igual que en el grupo control, no se presenta un patrón específico en sus valores, ni diferencias entre los grupos de edad, por lo tanto, presenta baja significancia estadística.

Tabla 12. Valores para la prueba de Límites de Estabilidad (LOS), en los tres grupos de edad, de los sujetos con pérdida auditiva y edad auditiva no compensada.

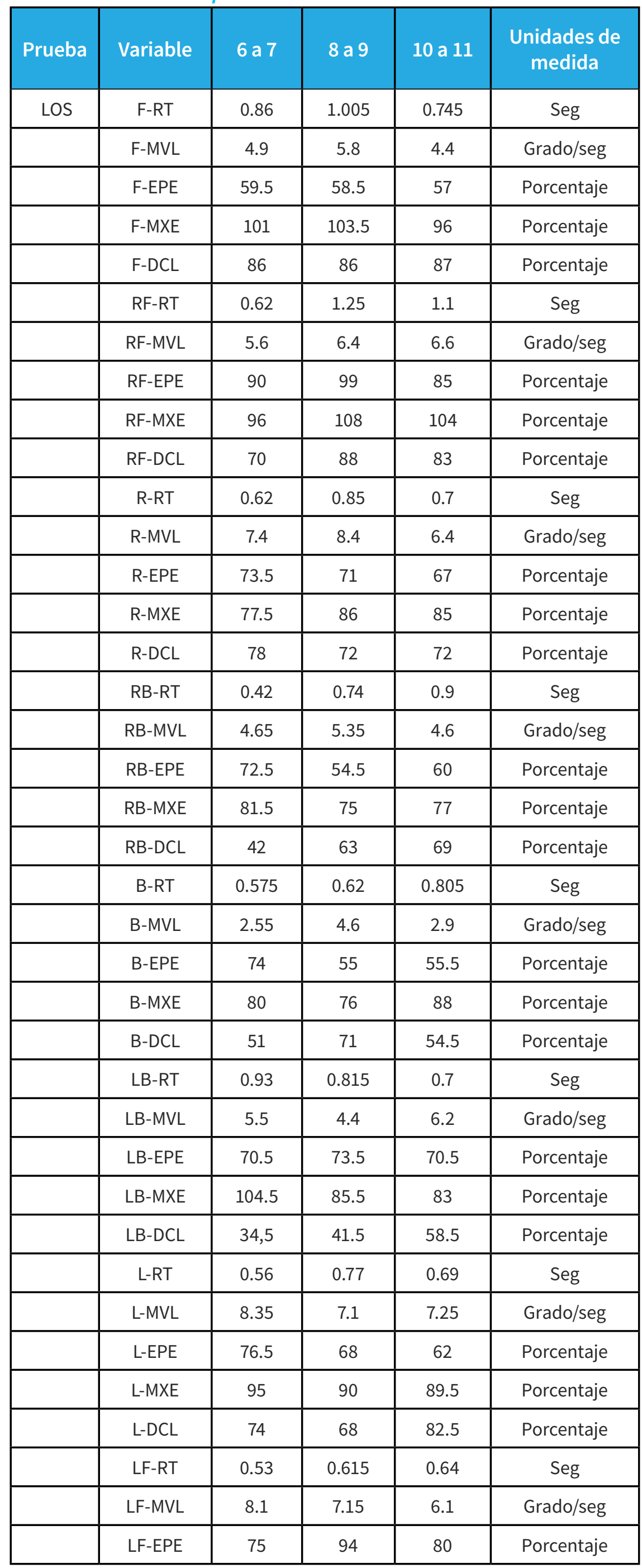




\begin{tabular}{|c|c|c|c|c|c|}
\hline Prueba & Variable & 6 a 7 & 8 a 9 & 10 a 11 & $\begin{array}{c}\text { Unidades de } \\
\text { medida }\end{array}$ \\
\hline & LF-MXE & 109.5 & 99.5 & 111 & Porcentaje \\
\hline & LF-DCL & 68 & 71 & 79 & Porcentaje \\
\hline & Comp-RT & 0.57 & 0.925 & 0.845 & Seg \\
\hline & Comp-MVL & 7 & 6 & 4.5 & Grado/seg \\
\hline & Comp-EPE & 72 & 71.5 & 68 & Porcentaje \\
\hline & Comp-MXE & 84 & 88 & 88 & Porcentaje \\
\hline & Comp-DCL & 60 & 61.5 & 70 & Porcentaje \\
\hline
\end{tabular}

Tiempo de reacción: adelante (F-RT), adelante a la derecha (RF-RT), derecha $(R-R T)$, atrás a la derecha (RB-RT), atrás (B-RT) atrás a la izquierda (LB-RT), izquierda (L-RT), adelante a la izquierda (LF-RT) y compilado (Comp-RT). Velocidad de movimiento: adelante (F-MVL), adelante a la derecha (RF$M V L)$, derecha (R-MVL), atrás a la derecha (RB-MVL), atrás (B-MVL) atrás a la izquierda (LB-MVL), izquierda (L-MVL), adelante a la izquierda (LF-MVL) y compilado (Comp-MVL). Punto de excursión mínimo (EPE) y máximo (MXE): adelante (F-EPE, F-MXE), adelante a la derecha (RF-EPE, RF-MXE), derecha (R-EPE, R-MXE), atrás a la derecha (RB-EPE, RB-MXE), atrás (B-EPE, B-MXE) atrás a la izquierda (LB-EPE, LB-MXE), izquierda (L-EPE, L-MXE), adelante a la izquierda (LF-EPE, LF-MXE) y compilado (Comp-EPE, Comp-MXE). Control direccional (DCL): adelante (F-DCL), adelante a la derecha (RF-DCL), derecha $(R-D C L)$, atrás a la derecha (RB-DCL), atrás (B-DCL) atrás a la izquierda (LB$D C L$ ), izquierda (L-DCL), adelante a la izquierda (LF-DCL) y compilado (Comp-

$D C L)$.

En los Límites de Estabilidad (LOS) del grupo de estudio, basados en los compilados, se observa que el tiempo de reacción en el grupo de niños entre seis y siete años es menor con respecto a los demás grupos, además, su velocidad de movimiento es mayor. Los valores de punto de excursión máximo y mínimo (MXE y EPE, respectivamente) se muestran aumentados en los niños entre 10 y 11 años, exponiendo que este grupo, estadísticamente hablando, eran quienes recorrían la mayor distancia entre las posiciones establecidas, e igualmente este grupo fue quien presentó un mejor control direccional, pues el desplazamiento producido está mejor orientado hacia el objetivo, este hallazgo se podría adjudicar a la presencia de reacciones reflejas en los niños de seis a siete años que requieren menor tiempo y cuentan con mayor velocidad, en cambio, en los niños de ocho a nueve años, la actividad ya es más voluntaria por lo que requiere mayor tiempo y menor velocidad, pero cuenta con mayor control direccional.

Es necesario destacar en este punto que, dos sujetos de este grupo de estudio presentaron caída durante la prueba, uno perteneciente al grupo de seis y siete años con caída en la posición adelante-derecha y otro del grupo de 10 y 11 años con caída hacia atrás-derecha.

\section{Comparación de los resultados entre el grupo control y el grupo de estudio.}

Para realizar la comparación, se obtuvieron valores estadísticos como la mediana y la desviación estándar, bajo el intervalo de confianza de 95\% estimado por medio de un método de remuestreo Bootstrap, y con un p-valor de la prueba de Wilcoxon. Se resalta además que la prueba de Wilcoxon se aplica posterior al juzgamiento de homogeneidad de varianzas mediante la prueba de Fligner teniendo en cuenta un nivel de significancia de 0.1 encontrando homogeneidad en todos los grupos para todas las variables.

Ahora, partiendo de los valores encontrados para cada prueba y en cada grupo de edad, se presentan las tablas 13, 14 y 15 en donde se especifican las variables en las que se presentaron diferencia estadísticamente significativa entre el grupo control y el grupo de estudio.

Tabla 13. Comparación entre los dos grupos en donde se rechaza la hipótesis nula de igualdad de medianas en los niños de seis y siete años

\begin{tabular}{|c|c|c|c|c|c|c|}
\hline \multirow{2}{*}{ Prueba } & \multirow{2}{*}{ Variable } & \multicolumn{2}{|c|}{ Grupo control } & \multicolumn{2}{|c|}{ Grupo de estudio } & \multirow{2}{*}{ Rechaza } \\
\hline & & Mediana & Desviación & Mediana & Desviación & \\
\hline WHT & $30-$ Izq & 50.00 & 30.289 .012 & 45.5 & 483.045 .892 & * \\
\hline \multirow[t]{7}{*}{ LOS } & F-EPE & 0.64 & 0.319929 & 0.595 & 0.2053452 & * \\
\hline & F-MXE & 0.95 & 0.1350729 & 1.01 & 0.2096624 & * \\
\hline & F-DCL & 0.81 & 0.0894258 & 0.86 & 0.4090945 & * \\
\hline & $\mathrm{R}-\mathrm{RT}$ & 0.86 & 0.3050025 & 0.62 & 0.1939071 & * \\
\hline & L-RT & 0.91 & 0.1888402 & 0.56 & 0.18138357 & * \\
\hline & LF-MXE & 0.9650 & 0.14559179 & 1.095 & 0.035939764 & * \\
\hline & Comp-DCL & 0.6750 & 0.06473138 & 0.6 & 0.122882057 & * \\
\hline
\end{tabular}


Con base en los datos obtenidos, se evidencia que los sujetos de estudio de seis a siete años se encuentran con desequilibrio en la distribución del peso corporal en sus extremidades en la flexión a 30, pues se encuentran descargando mayor peso sobre la pierna derecha, esto estaría ligado a la dominancia de la pierna derecha que presentan los niños. En cuanto a la prueba de Límites de Estabilidad (LOS), destaca la diferencia en el control direccional, por lo que se les dificulta más alcanzar la posición objetivo, además el tiempo de excursión mínimo se encuentra por debajo de la normativa, por el contrario, en los tiempos de reacción hacia la derecha y hacia la izquierda presentan mejor rendimiento, al igual que para los puntos máximos de excursión hacia el frente y hacia el frente a la izquierda.

Hay que destacar que, en este grupo de edad, se presentó caída en un sujeto en la prueba de Límites de Estabilidad, en la variable de al frente-derecha, mientras que este suceso no ocurrió en ningún sujeto del grupo control.

Tabla 14. Comparación de medianas entre niños entre ocho y nueve años de ambos grupos

\begin{tabular}{|c|c|c|c|c|c|c|}
\hline \multirow{2}{*}{ Prueba } & \multirow{2}{*}{ Variable } & \multicolumn{2}{|c|}{ Grupo control } & \multicolumn{2}{|c|}{ Grupo de studio } & \multirow{2}{*}{ Rechaza } \\
\hline & & Mediana & Desviación & Mediana & Desviación & \\
\hline CTSIB & Vel Osc_Foam-OA & 0.9 & 0.23208641 & 1.2 & 0.2607681 & * \\
\hline \multirow{7}{*}{ LOS } & F-EPE & 0.64 & 0.318940388 & 0.585 & 0.305379545 & * \\
\hline & F-MXE & 1 & 0.38393973 & 1.035 & 0.310188115 & * \\
\hline & RF-EPE & 0.64 & 0.175100704 & 0.99 & 0.252843825 & * \\
\hline & RF-MXE & 1.02 & 0.129708747 & 1.08 & 0.085264295 & * \\
\hline & RF- DCL & 0.73 & 0.183847763 & 0.88 & 0.011401754 & * \\
\hline & R-MVL & 4.6 & 26.817 .761 & 8.4 & 258.205 .345 & * \\
\hline & LB-EPE & 0.58 & 0.223314197 & 0.735 & 0.124579292 & * \\
\hline & LB-DCL & 0.63 & 0.170568431 & 0.415 & 0.257248259 & * \\
\hline & L-MVL & 5.7 & 171.919 .779 & 7.1 & 246.536 .001 & * \\
\hline & LF-EPE & 0.76 & 0.193837759 & 0.94 & 0.267781005 & * \\
\hline
\end{tabular}

F-EPE: punto mínimo de excursión, adelante. F-MXE: punto máximo de excursión, adelante. RF-EPE: punto mínimo de excursión, derecha al frente. RF-MXE: punto máximo de excursión, derecha al frente. RF- DCL: control direccional, derecha al frente. R-MVL: velocidad de movimiento, derecha. LB-EPE: punto mínimo de excursión, izquierda y atrás. LB-DCL: control direccional, izquierda y atrás. L-MVL: velocidad de movimiento, izquierda. LF-EPE: punto mínimo de excursión,

izquierda al frente

En la tabla 14, se revelan las variables que presentan diferencia significativa entre el grupo control y el grupo de estudio para los niños entre ocho y nueve años; tales variables son: Vel Osc_Foam-OA (velocidad de oscilación en la condición de superficie inestable con ojos abiertos) que pertenece a la prueba de Integración Sensorial y F-EPE (punto mínimo de excursión - adelante), F-MXE (punto máximo de excursión - adelante), RF-EPE (punto mínimo de excursión - adelantederecha), RF-MXE (punto máximo de excursión - adelante-derecha), RFDCL (control direccional - adelante-derecha), LB-EPE (punto mínimo de excursión - atrás izquierda), LB-DCL(control direccional - atrásizquierda), L-MVL (velocidad de movimiento - izquierda) y LF-EPE (punto mínimo de excursión - adelante-izquierda) pertenecientes a la prueba de Límites de Estabilidad.

Tabla 15. Comparación de medianas entre niños de 10 y 11 años de ambos grupos
Con esta información se puede decir que, los niños de ocho y nueve años del grupo de estudio, presentan dificultad para mantener el equilibrio cuando se les retira el apoyo somatosensorial, ubicándolos en la plataforma inestable aún con ojos abiertos; en cuanto a los límites de estabilidad, se evidencia dificultad únicamente en el control direccional hacia atrás a la izquierda; pues el restante de variables de dicha prueba que se agrupan en los puntos de excursión mínima y máxima y la velocidad del movimiento, obtuvieron mejor rendimiento en los sujetos en estudio, esto puede atribuirse a que se encuentran vinculados a una institución que aparte de ofrecer rehabilitación auditiva y del lenguaje, presta el servicio de terapia ocupacional en donde se están fortaleciendo habilidades para el equilibrio, a diferencia de los niños del grupo control quienes no reciben ningún tipo de apoyo terapéutico.

\begin{tabular}{|c|c|c|c|c|c|c|}
\hline \multirow{2}{*}{ Prueba } & \multirow{2}{*}{ Variable } & \multicolumn{2}{|c|}{ Grupo Control } & \multicolumn{2}{|c|}{ Grupo de estudio } & \multirow{2}{*}{ Rechaza } \\
\cline { 3 - 7 } & & Mediana & Desviación & Mediana & Desviación & \\
\hline \multirow{2}{*}{ LOS } & F-DCL & 0.82 & 0.406650697 & 0.87 & 0.335499627 & $*$ \\
\cline { 2 - 7 } & RF- DCL & 0.72 & 0.303362113 & 0.83 & 0.0589067059 & * \\
\hline
\end{tabular}


En la tabla 15, se puede observar que las variables que tuvieron diferencia entre el grupo control y el grupo de estudio son: F-DCL (control direccional, adelante) y RF-DCL (control direccional, adelantederecha), ambas pertenecientes a la prueba de Límites de Estabilidad (LOS), lo que informa que el grupo de 10 y 11 años con pérdida auditiva, presentaron mejor desempeño en el control direccional hacia adelante y adelante a la derecha, predominantemente; sin embargo, hay que recordar que para el presente grupo de edad se presentó una caída en los Límites de Estabilidad (LOS) hacia atrás a la derecha, por lo que ese sujeto tendría latente el riesgo de caída en actividades que le exijan esta dirección, acotando que en los infantes del grupo control no ocurrió dicho evento en ninguna dirección.

\section{Terapia ocupacional}

A continuación, en la tabla 16 se exponen los resultados por grupos de edad y por variables de las pruebas del equilibrio que se les realizaron a los participantes del grupo de estudio por parte de Terapia Ocupacional en la Fundación CINDA.

Tabla 16. Resultados de terapia ocupacional encontrados en el grupo de estudio en pruebas del equilibrio

\begin{tabular}{|c|c|c|c|c|c|c|c|c|c|}
\hline \multirow{2}{*}{ Variable } & \multicolumn{4}{|c|}{6 a 7} & \multicolumn{3}{c|}{8 a 9 } & \multicolumn{3}{c|}{10 a 11} \\
\cline { 2 - 11 } & $\mathrm{P}$ & $\mathrm{D}$ & $\mathrm{N}$ & $\mathrm{P}$ & $\mathrm{D}$ & $\mathrm{N}$ & $\mathrm{P}$ & $\mathrm{D}$ & $\mathrm{N}$ \\
\hline Mov ocular automático & 0 & 3 & 1 & 0 & 2 & 4 & 0 & 3 & 3 \\
\hline Mov ocular voluntario & 0 & 3 & 1 & 0 & 3 & 3 & 0 & 3 & 3 \\
\hline Prono-extensión & 1 & 3 & 0 & 0 & 3 & 3 & 0 & 3 & 3 \\
\hline Control postural reactivo & 0 & 3 & 1 & 0 & 3 & 3 & 0 & 0 & 6 \\
\hline Control postural anticipatorio & 1 & 2 & 1 & 0 & 5 & 1 & 0 & 4 & 2 \\
\hline Extensión protectora & 0 & 1 & 3 & 0 & 0 & 6 & 0 & 0 & 6 \\
\hline Seguridad gravitacional & 0 & 0 & 4 & 0 & 1 & 5 & 0 & 0 & 6 \\
\hline Integración Visomotriz (VMI) & 0 & 0 & 4 & 0 & 2 & 4 & 0 & 1 & 5 \\
\hline Coordinación motriz & 0 & 1 & 3 & 0 & 1 & 5 & 0 & 1 & 5 \\
\hline
\end{tabular}

$P=$ Pobre, $D=$ Dificultad, $N=$ Normal

Se evidencia que, por grupos de edad, los niños de seis a siete años son los que presentan mayores deficiencias en estas pruebas, pues en los movimientos oculares automáticos, en los movimientos oculares voluntarios y el control postural reactivo, tres niños puntuaron con dificultad y sólo uno en normalidad. Para la prono-extensión, un niño presentaba pobre habilidad y los otros tres presentaban dificultad. En cuanto al control postural anticipatorio, un niño se encontraba en pobre habilidad, dos con dificultad y uno en rango de normalidad. Pasando a la extensión protectora y la coordinación motriz, sólo un infante presentaba dificultad mientras que los tres restantes se encontraban en normalidad y respecto a seguridad gravitacional y la integración viso motriz, todos los niños se encontraron con habilidades normales.

El grupo etario que sigue en orden de dificultad es el de los niños entre ocho y nueve años, en donde se presentaban movimientos oculares automáticos e integración viso motriz con dificultad en dos niños y en donde cuatro se encontraron con valores normales, en lo que concierne a los movimientos oculares voluntarios, prono-extensión y control postural reactivo tres niños cursaban con dificultad y tres en normalidad. En relación con el control postural anticipatorio, cinco sujetos presentaban dificultad y uno estaba en el rango de normalidad; en seguridad gravitacional y coordinación motriz, un niño presentaba dificultad mientras que los otros cinco tenían esas habilidades normales y, por último, en extensión protectora todos los niños se encontraban en normalidad.

Finalizando con los niños de 10 a 11 años, quienes son los que presentan menos dificultades en las variables evaluadas, se encuentra que, en cuanto a movimiento ocular automático, movimiento ocular voluntario y prono-extensión, tres niños se clasifican con dificultad y los tres restantes en normalidad. En referencia al control postural reactivo, la extensión protectora y la seguridad gravitacional todos los sujetos se encuentran con las habilidades normales; el control postural anticipatorio se presenta con dificultad en cuatro niños y normalidad en dos, y, por último, en relación con la integración viso motriz y la coordinación motriz, sólo en un sujeto se encuentra dificultad y normalidad en los cinco restantes.

A nivel global, lo que se observa es que, en los tres grupos, la variable con mayor dificultad fue el control postural anticipatorio denotando que, en general, los niños tenían dificultad para iniciar correctamente un movimiento o que tenían peor capacidad para aplicar acciones correctivas/preventivas para prevenir una caída y la que reportó menor dificultad es la seguridad gravitacional, indicando menor ansiedad global cuando el sistema vestibular detecta cambios gravitacionales.

Es menester mostrar en este punto, la relación concordante entre los datos arrojados en la posturografía y los obtenidos por evaluación perceptual de Terapia Ocupacional, pues en ambas pruebas, los grupos con más diferencias en cuanto a dificultades o deficiencias fueron los dos primeros, es decir, los de seis a siete y de ocho a nueve, así como se refleja que el grupo de 10 a 11 años quienes en la posturografía no mostraron diferencias negativas estadísticamente significativas, igualmente en la evaluación perceptual de Terapia Ocupacional fue el grupo de infantes con menos dificultades.

Así mismo, se evidenció que el grupo en estudio tuvo mejor desempeño en algunas de las variables de la posturografía en la prueba de Límites de Estabilidad, posiblemente atribuible al apoyo terapéutico recibido, en donde se fortalecen habilidades para el mantenimiento y/u optimización del equilibrio mediante enfoque de Integración Sensorial. 


\section{Posturografía en niños entre 6 -11 años con edad auditiva no compensada}

\section{Discusión}

El equilibrio corporal depende de información sensorial que da cuenta de cómo se mueve el cuerpo y del estado actual de estabilidad. Esta información proviene del sistema visual (movimiento relativo ojos-ambiente), vestibular (aceleración de la cabeza en el espacio) y propioceptivo (rotación de las articulaciones y cambios en la presión cutánea), lo que sugiere que el cuerpo es un sistema dinámico capaz de ajustarse a medida que los canales de información hacen su función. Según los valores obtenidos durante la prueba de Integración Sensorial (CTSIB) tanto para el grupo control como para el grupo de estudio, a medida que aumenta la dificultad de la prueba, en las condiciones que requieren el retiro del apoyo visual y/o somatosensorial, los valores de la velocidad de oscilación aumentan de manera directamente proporcional, así mismo, se observa disminución en los rangos de los valores a medida que aumenta la edad. Dicha disminución se atribuye a la neuromaduración y las habilidades adquiridas.

Lo anterior concuerda con lo expuesto por (Cardoso, 2018) y (Daley, 1982) cuando en sus investigaciones sostienen que la estabilidad tiende a aumentar con la edad y a disminuir con la manipulación sensorial, asimismo, reportan que la maduración sensorial se produce primero en el sistema visual, luego en el sistema propioceptivo y por último en el sistema vestibular. Se observa también que en la Alineación del Centro de Gravedad (COG) en el grupo control, el valor máximo corresponde al grupo de seis y siete años de edad y el menor valor, corresponde a los niños de ocho, nueve, diez y once años de edad, lo que se atañe al hecho de que los niños más grandes, poseen una lateropulsión menos evidente y menor riesgo de caída de altura corporal. De acuerdo con lo anterior, (Gouléme, 2018), en su estudio pediátrico multicéntrico, llegaron a la conclusión de que el control postural analizado con velocidad media del centro de presión (CoP) y el Índice de Inestabilidad Postural (PII), mejoran con la edad, y esto se relaciona con el proceso de maduración, el cual aún no se ha completado a los 16.08 años y refieren que incluso a esa edad continúa el proceso de neuromaduración.

En cuanto a la Alineación del Centro de Gravedad (COG) se reporta estudios como en el de (Daley, 1982), que el balanceo del centro de gravedad a lo largo de la vida tiene unos valores que se asemejan a una función con curva en forma de $U$, en donde los niños y los adultos mayores tienen valores del COG semejantes, aunque con un balanceo superior al de los adultos. En el caso de los niños, este comportamiento es resultado de la inmadurez del SNC, mientras que, en los adultos mayores, esto se da por falencias en la integración de estímulos visuales, vestibulares y somatosensorial asociados con la edad.

Las diferencias presentadas entre los niños del estudio en cuanto a lateralidad en los Límites de Estabilidad (LOS) pueden deberse a la dominancia de uno u otro hemisferio cerebral, respecto a esta prueba (Reyes, 2018), descubrieron que en adultos con patología neuronal, se observaban dificultades hacia un lado u otro y se encontró una relación con la lateralidad del sujeto, de lo cual se puede inferir que es posible que la dominancia cerebral tenga alguna influencia sobre el control postural.

Entre las diferencias estadísticamente significativas encontradas en la posturografía del grupo de estudio frente al grupo control destacan la dificultad en la prueba de Límites de Estabilidad (LOS) en el control direccional y el punto mínimo de excursión hacia adelante, presente tanto en los niños de seis a siete años como en los de ocho y nueve años, es decir, presentaban dificultad para dirigirse con precisión hacia el punto objetivo y recorrían una menor distancia en el primer intento por ir hacia adelante.
Frente a este hallazgo, no fue posible encontrar literatura en niños, sin embargo, (Bujang, 2013) refieren que en adultos usuarios de Implante Coclear frente a controles sanos, no presentaron diferencias significativas en estas variables de Límites de Estabilidad (LOS), lo que era atribuible a una respuesta de adaptación vestibular frente a la novedad del implante por parte del grupo en estudio, lo que estaría en oposición a lo reportado con anterioridad que podría ser explicado por la diferencia de edad de los sujetos y por ende, diferentes habilidades adquiridas.

Por otra parte, el grupo etario de ocho a nueve años de los niños en estudio, presentaron diferencia significativa en la prueba de Integración Sensorial, en la tercera condición que exige, una plataforma inestable y ojos abiertos, la diferencia en dicha prueba ya fue expuesta por algunos autores, entre ellos (Andrew Kelly, 2018) y (Licameli G, 2009), en este último el 39\% de la población usuaria de implante coclear de su estudio tuvo hallazgos anormales que indican déficit en la organización sensorial.

En el presente estudio se presentaron dos caídas en los ejes derecha-anterior y derecha posterior, ambas en niños con pérdida auditiva, mientras que en los niños del grupo control no se presentó este tipo de situaciones. Igualmente, se encontró que, en estos niños, en la prueba de Distribución de peso (WHT), las cargas no se distribuían uniformemente a $90^{\circ}$ de flexión, y este es un indicador indirecto de la integridad y buena coordinación en este tipo de pruebas. Este tipo de situaciones se vieron reflejadas en otros estudios, por ejemplo, (Ramírez, 2016), analizó los valores posturográficos entre caedores recurrentes y sujetos control, en donde los sujetos caedores recurrentes, a medida que aumentaba la flexión de las rodillas, apoyaban su peso predominantemente en una de las extremidades, al punto de que estas diferencias alcanzaron diferencia estadística (p. 117).

Continuando, la baja diferencia de la prueba de Distribución de Peso entre los grupos de edad y entre el grupo control frente al grupo de estudio (WHT) se debió a que esta prueba tiene un mayor porcentaje de sensibilidad en sujetos con afecciones articulares, que se encuentran débiles o con un gran dolor como para apoyar gran parte de su peso corporal en una sola articulación (Bascuas Rodríguez, 2013), por tanto, se explica la baja significancia de esta prueba en el estudio.

El enfoque de integración sensorial como terapia de mantenimiento y mejoramiento del equilibrio recibido en los niños del estudio con pérdida auditiva, tiene como finalidad hacer un entrenamiento para integrar y organizar las sensaciones provenientes de los sentidos (Del Moral Orro, 2013). En este orden de ideas, el objetivo de este entrenamiento es educar a los niños para producir respuestas adaptativas que les permitan hacer frente a situaciones (naturales o artificiales como en la posturografía) adversas del equilibrio. Durante este entrenamiento, es posible rastrear el desempeño de los niños en términos de edad motriz (equiparable al concepto de edad auditiva) y llevar a cabo un trabajo que se encamine a nivelar la edad motriz y la edad cronológica.

En atención a lo anterior, cabe mencionar que los niños con pérdida auditiva que asistían a terapia ocupacional, se encontraban nivelando su edad motriz, esto explicaría el desempeño en la prueba de Límites de Estabilidad (LOS), pues se hallaron pocas diferencias negativas estadísticamente significativas con respecto a los niños del grupo control y otras variables que por el contrario reflejan un mejor rendimiento frente a este grupo. No obstante, la variable de menor desempeño en este grupo de niños fue la de control postural anticipatorio, a lo que se adjudicaría las caídas presentadas por dos niños, debido a la dificultad para aplicar medidas posturales para prevenir o corregir la posibilidad de una caída. 


\section{Conclusiones}

La posturografía es un batería de pruebas para la evaluación del equilibrio que da cuenta del neurodesarrollo de los niños, pues con el paso de la edad se evidencia cómo estos valores cambian a lo largo del tiempo por acción de la neuromaduración.

Se encontró que los niños más pequeños del grupo de estudio, es decir, los de seis a siete y ocho a nueve años, exhiben diferencias estadísticamente significativas que reflejan dificultad en la prueba de Límites de Estabilidad (LOS) en cuanto al control direccional y en el punto mínimo de excursión hacia al frente. Además, durante la realización de la misma prueba, dos de los sujetos de estudio presentaron caída, situación que no ocurrió en ningún caso en el grupo control.

Por otra parte, los sujetos de estudio presentaron mejor rendimiento en algunas variables de la prueba de Límites de Estabilidad (LOS), como en los puntos mínimos y máximos de excursión y la velocidad de movimiento, estos desempeños fueron atribuibles a la intervención terapéutica que reciben por Terapia Ocupacional en la Fundación CINDA, a diferencia de los niños del grupo control que no reciben ningún tipo de apoyo terapéutico; esta afirmación se sustenta en el hecho de que durante la terapia se trabaja la seguridad gravitacional, el control postural y la coordinación motriz mediante enfoque de Integración Sensorial, estos son procesos esenciales para presentar mejor rendimiento en estas variables.

Respecto a la prueba de Integración Sensorial (CTSIB), sólo se presentó diferencia significativa en la condición que requiere superficie inestable y ojos abiertos en los infantes del grupo etario de ocho a nueve años. Por lo que concierne a la prueba de Distribución de Peso (WHT), sólo se encontró diferencia significativa en la variable de flexión a $30^{\circ}$ en los niños de seis a siete años, en donde se evidencia que distribuyen mayor peso a la pierna derecha y concuerda con su dominancia de extremidad.

La prueba de Distribución de Peso (WHT), mostró baja diferencia estadística entre los grupos por edad y a su vez, entre el grupo control y el grupo de estudio; sin embargo, esta prueba es un importante predictor de caídas, sobre todo en poblaciones con indicadores morfológicos de desigualdad de distribución del peso corporal, por ejemplo, en desórdenes relacionados con afecciones músculoesqueléticas o neurológicas, los cuales no se encontraron durante el estudio en la anamnesis o en la revisión de historias clínicas.

Los resultados de la evaluación del equilibrio realizada por Terapia Ocupacional concuerdan con los datos obtenidos por grupos de edad en la posturografía, pues refleja que los grupos con más diferencias en cuanto a dificultades o deficiencias fueron los dos primeros, es decir, los de seis a siete y de ocho a nueve. El grupo de 10 a 11 años quienes en la posturografía no mostraron diferencias negativas estadísticamente significativas al igual que en la evaluación perceptual de Terapia Ocupacional fue el grupo de infantes con menos dificultades, lo que deja en evidencia que esta valoración es eficiente y útil para el monitoreo constante de los avances de intervención.

Igualmente, con los resultados obtenidos en esta investigación, en donde se evidencian las dificultades en cuanto a límites de estabilidad e integración sensorial de la población en estudio, se confirma la importancia de que los niños con deficiencia auditiva reciban apoyo terapéutico para el reforzamiento, mantenimiento y optimización del equilibrio y así su rehabilitación sea integral, ofreciendo una mayor calidad de vida.
Se sugiere la creación de un software en la posturografía que sea más amigable para la población pediátrica, en donde los ejercicios a realizar se adapten a sus intereses y su aplicación sea más agradable y didáctica. Por ejemplo, en la prueba de Límites de Estabilidad (LOS), para el alcance de las posiciones objetivo, se pueden generar actividades de relacionar o lograr el encuentro entre complementos, como el ave llegando al nido o el perro buscando su casa.

Por otra parte, se recomienda ampliar la población de estudio en próximos trabajos de investigación que ahonden en el tema y así aportar al análisis estadístico del mismo.

Finalmente, para futuras investigaciones se propone estudiar el desempeño entre niños que reciben el apoyo terapéutico integral, con grupo interdisciplinario, en donde juega un papel fundamental Fonoaudiología y Terapia Ocupacional frente a los que no reciben este apoyo.

\section{Referencias}

Hamilton, S., Yoo, J., Hammond, A., Read, N., Venkatesan, V., Franklin, J., Badhwar, A. (Oct de 2008). Microvascular changes in radiationinduced oral mucositis. J Otolaryngol Head Neck Surg, 37(5), 730-737.

Zhang, L., Huang, Z., Wu, H., Chen, W., \& Huang, Z. (Dic de 2014). Effect of swallowing training on dysphagia and depression in postoperative tongue cancer patients. Eur J Oncol Nurs, 18(6), 626-629.

Lazarus, C. (Jun de 2009). Effects of chemoradiotherapy on voice and swallowing. Curr Opin Otolaryngol Head Neck Surg, 17, 172-178.

Frowen, J., Hughes, R., \& Skeat, J. (2019). The prevalence of patientreported dysphagia and oral complications in cancer patients. Supportive Care in Cancer, doi: https://doi.org/10.1007/s00520-01904921-y.

Siu, L., Waldron, J., Chen, B., Winquist, E., Wright, J., Nabid, A., .. . Johnson, A. (Dic de 2016). Effect of Standard Radiotherapy With Cisplatin vs Accelerated Radiotherapy With Panitumumab in Locoregionally Advanced Squamous Cell Head and Neck Carcinoma: A Randomized Clinical Trial. JAMA oncology, 3(2), 220-226.

Alvarez, C., Llorente, J., Gutierrez, V., Fernandez, H., Guervos, M., Suarez, C., \& Hermen, M. (2007). Recidivas en el cáncer escamoso de cabeza y cuello. Acta Otorrinolaringol Esp, 58(4), 156-163.

Hutcheson, K., Lewin, J., Barringer, D., Lisec, A., Gunn, B., Moore, M., \& Holsinger, C. (2012). Late Dysphagia After Radiotherapy-Based Treatment of Head and Neck Cancer. Cancer, 118(23), doi: https://doi. org/10.1002/cncr.27631.

Suárez, L., Puerto-García, S., Rodríguez-Moreno, L., \& Ramírez-Moreno, J. (2017). La crisis del sistema de salud colombiano: una aproximación desde la legitimidad y la regulación. Rev. Gerenc. Polit. Salud, Bogotá, Colombia, 16(32), 34-50.

Cámpora, H., \& Falduti, A. (2012). Evaluación y tratamiento de las alteraciones de la deglución. Rev Am Med Resp, 3(1), 98-107.

Wheeler-Hegland, K., Ashford, J., Frymark, T., McCabe, D., Mullen, R., Musson, N., ... Schooling, T. (2009). Evidence-based systematic review: Oropharyngeal dysphagia behavioral treatments. Part IIImpact of dysphagia treatment on normal swallow function. Journal of rehabilitation research and development, 46(2), 185-194.

Cárcamo. (2018). Epidemiología y generalidades del tumor de cabeza y cuello. Revista Médica Clínica Las Condes, 29(4), 387-492. doi: https:// doi.org/10.1016/j.rmclc.2018.06.009

Liga Contra el Cáncer. (01 de 01 de 2016). Liga contra el cáncer seccional Bogotá. Recuperado el 18 de 06 de 2019, de http://www. ligacontraelcancer.com.co/cancer-de-cabeza-y-cuello/ 
American Cancer Society, NHC. (2015). American Cancer Society. Recuperado el 5 de Octubre de 2016, de Guía sobre Radioterapia: https://www.cancer.org/es/tratamiento/tratamientos-y-efectossecundarios/tipos-de-tratamiento/radioterapia.html

Greenblatt, D., Sippel, R., Leverson, G., Frydman, F., Schaefer, S., \& Chen, H. (2009). Thyroid Resection Improves Perception of Swallowing Function. World Journal of Surgery, 33, 255-260. doi: https://doi. org/10.1007/s00268-008-9837-9

Nickel, B., Tan, T., Cvejic, E., Baade, P., McLeod, D., Pandeya, N., . . . Jordan, S. (17 de 01 de 2019). Health-Related Quality of Life After Diagnosis and Treatment of Differentiated Thyroid Cancer and Association With Type of Surgical Treatment. JAMA Otolaryngology-Head \& Neck Surgery, e1-e8. doi: https://doi.org/10.1001/jamaoto.2018.3870.

Cadena, E., Guerra, R., \& Pérez-Mitchell, C. (Julio-Septiembre de 2014). Cirugía Robótica Transoral (TORS), en el manejo de lesiones neoplásicas de cabeza y cuello. Revista Colombiana de Cancerología, 18(3), 128-136. doi: https://doi.org/10.1016/j.rccan.2014.06.003

Carcamo, M. (Ago de 2018). Epidemiología y generalidades del tumor de cabeza y cuello. Revista Médica Clínica Las Condes, 29(4), 387-492.

Liga contra el cáncer. (01 de 01 de 2016). Liga contra el cáncer seccional Bogotá. Recuperado el 18 de 06 de 2019, de http://www. ligacontraelcancer.com.co/cancer-de-cabeza-y-cuello/

American Cancer Society, NHC. (2015). Recuperado el 5 de Octubre de 2016, de Una guía sobre Radioterapia: http://www.cancer.org/acs/ groups/cid/documents/webcontent/003027-pdf.pdf

Greenblatt, D., Sippel, R., Leverson, G., Frydman, F., Schaefer, S., \& Chen, H. (2009). Thyroid Resection Improves Perception of Swallowing Function. World Journal of Surgery, 33, 255-260. doi:https://doi. org/10.1007/s00268-008-9837-9

Nickel, B., Tan, T., Cvejic, E., Baade, P., McLeod, D., Pandeya, N., . . Jordan, S. (17 de jan de 2019). Health-Related Quality of Life After Diagnosis and Treatment of Differentiated Thyroid Cancer and Association With Type of Surgical Treatment. JAMA Otorinolaryngology - Head \& Neck Surgery, 231-238. doi: https://doi.org/10.1001/jamaoto.2018.3870

Cadena, E., Guerra, R., \& Pérez-Mitchell, C. (Julio-Septiembre de 2014). Cirugía Robótica Transoral (TORS), en el manejo de lesiones neoplásicas de cabeza y cuello. Revista Colombiana de Cancerología, 18(3), 128-133. doi:https://doi.org/10.1016/j.rccan.2014.06.003

European Society for Medical Oncology. (01 de 01 de 2015). Fundación contra el cáncer. Recuperado el 18 de 06 de 2019, de ESMO: ESMOACF-Cancer-de-Cabesa-y-Cuello-Guia-para-pacientes.pdf

Smeele, L. (2014). Oral, oropharyngeal and nasopharyngeal cancer: intervention approaches. En E. Ward, \& C. Van As-Brooks, Head and neck cancer: treatment, rehabilitation, and outcomes ( ed., págs. 103120). San Diego, California: Plural Publishing.

Denaro, N., Merlano, M., \& Russi, E. (Sep de 2013). Dysphagia in Head and Neck Cancer Patients: Pretreatment Evaluation, Predictive Factors, and Assessment during Radio-Chemotherapy, Recommendations, Clinical and Experimental Otorhinolaryngology., 6(3), 117126. doi:https://www.e-ceo.org/journal/view.php?doi=10.3342/ ceo.2013.6.3.117

Logemann, J., Pauloski, B., Rademaker, A., \& Colangelo, L. (1 de Mayo de 1997). Speech and swallowing rehabilitation for head and neck cancer patients. Oncology (Williston Park), 11(5), 659-664.

Pauloski, B. (Nov de 2008). Rehabilitation of Dysphagia Following Head and Neck Cancer. Physical Medicine and Rehabilitation Clinic of North America, 19(4), 889-928. doi:doi: https://doi.org/10.1016/j. pmr.2008.05.010.

Santini, L., Robert, D., Lagier, A., Dessi, P., \& Fakhry, N. (Junio de 2015). A videofluoroscopic study comparing severe swallowing disorders in patients treated surgically or with radiation for oropharyngeal cancer. International Journal of Oral and Maxillofacial Surgery, 44(6), 705-709. doi:https://www.ijoms.com/article/S0901-5027(15)00044-2/fulltext
Portas, J., Pereira, C., Perissato, E., Dos Santos, D., Sampaio, A., Aparecido, R., \& Brandao, A. (Ene-Feb de 2011). Swallowing after non-surgical treatment (radiation therapy / radiochemotherapy protocol) of laryngeal cancer. Brazilian Journal of Otorhinolaryngology, 77(1), 96101.

Szczesniak, M., Maclean, J., Zhang, T., Graham, P., \& Cook, I. (Nov de 2014). Persistent Dysphagia after Head and Neck Radiotherapy: A Common and Under-reported Complication with Significant Effect on Non-cancer-related Mortality. Clinical Oncology (Royal College of Radiologist), 26(11), 697-703. doi:doi: https://doi.org/10.1016/j. clon.2014.08.009

Shaw, S., \& Martino, R. (Dec de 2013). The normal swallow: muscular and neurophysiological control. Otolaryngology Clinics of North America, 46(6), 937-956. doi: https://doi.org/10.1016/j.otc.2013.09.006.

Joung, S., Woo, K., Beom, S., Hwa, J., \& Kyu, M. (Oct de 2015). Voluntary Cough and Swallowing Function Characteristics of Acute Stroke Patients Based on Lesion Type. Archives of Physical Medicine and Rehabilitation, 96(10), 1866-1872. doi:https://doi.org/10.1016/j. apmr.2015.06.015

Lyndell, K. (2014). Radiation and Chemotherapy. En E. Ward, \& C. Van as-brooks, Head and neck cancer: treatment, rehabilitation, and outcomes (Second Edition ed., págs. 63-102). San Diego, California: Plural Publishing.

Martínez, M., \& Bellido, D. (2003). Nutrición y disfagia. Fisioterapia, 25(5), 306-310. doi:https://doi.org/10.1016/S0211-5638(03)73069-7

Lazarus, C., Ward, E., \& Yiu, E. (2014). Speech and Swallowing following oral, oropharyngeal and nasopharyngeal cancers. En E. Ward, \& C. Van As-Brooks, Head and Neck Cancer: Treatment, Rehabilitation, and Outcomes (págs. 173-240). San Diego, California: Plural Publishing.

Biblioteca Virtual de Salud. (15 de Mayo de 2018). Descriptores en Ciencias de la Salud. Obtenido de DeCS: http://decs.bvs.br/cgi-bin/wxis1660. exe/decsserver/

Vargas, M. (Abril-Junio de 2018). Repercusiones sociales de la disfagia. Revista de Logopedia, Foniatría y Audiología, 38(2), 77-83. doi:https:// doi.org/10.1016/j.rlfa.2017.10.003

Vidal, F. (2018). Hacia una fenomenología del síndrome de cautiverio. Rev. Asoc. Esp. Neuropsiq., 38(133), 45-73. doi: https://doi.org/10.4321/ S0211-57352018000100003

Orphadata. (17 de Mayo de 2018). Clasifications of rare diseases: hierarchical representation. Obtenido de http://www.orphadata.org/ cgi-bin/inc/product3.inc.php

International Dysphagia Diet Standardisation Initiative. (10 de October de 2016). Complete IDDSI framework and descriptors. Obtenido de iddsi.org/translation: https://iddsi.org/wp-content/uploads/2018/05/ Marco y descriptores de la IDDSI FOR REVIEW.pdf

Mansueto, A., Aguiar, S., Oliveira, e., Caseiro, L., \& Teixeira, A. (2016). Frequência e fatores associado à disfagia após acidente vascular cerebral. CoDAS, 28(1), 66-70. doi:https://doi.org/10.1590/2317$\underline{1782 / 20162015072}$

Vargas, M. (2015). Algoritmo de transformación de consistencia como estrategia de abordaje fonoaudiológico. . Rev. Col. Reh., 14(1), 22 - 27.

Vargas, M. (30 de junio de 2017). Repercusiones sociales de la disfagia: revisión sistemática. Revista Colombiana de Rehabilitación, 16(1), 32 39. doi:https://doi.org/10.30788/RevColReh.v16.n1.2017.63

Pacheco-Hernández, A., Ramos Villegas, Y., Padilla Zambrano, H., Mendoza Florez, R., Quintana Pájaro, L., López Cepeda, D., . . Moscote Salazar, L. R. (2017). Síndrome de Locked-In: Reporte de Caso. Revista Ecuatoriana de Neurología, 26(3), 301-305.

Khanna, K., Verma, A., \& Richard, B. (December de 2011). The locked-in syndrome: Can it be unlocked? Journal of Clinical Gerontology and Geriatrics, 2(4), 96-99.

Lujan Ramos, V. P., Monterrosa-Salazar, E., \& Polo-Verbel, L. (2011). Síndrome de enclaustramiento: a propósito de un caso. Revista Ciencias Biomédicas, 2(1), 116-120. 
Arroyo-Aljaro, R., González-Viejo, M., \& Rovira, A. (2008). Síndrome de locked-in. Una mirada a su tratamiento y pronóstico. Rehabilitación, 42(2), 92-95. doi:https://doi.org/10.1016/S0048-7120(08)73620-X

Flores, K., Berretin-Felix, G., Beltrati, M., \& Queiroz, I. (ABRIL-JUNIO de 2009). Avaliação miofuncional orofacial - Protocolo MBGR. CEFAC, 11(2), 237-255.

Furkim, A., \& Freitas, A. (2008). Eficácia da fonoterapia em disfagia neurogênica usando a escala funcional de ingestão por via oral (FOIS) como marcador. Rev CEFAC, 10(4), 503-512. doi:https://doi. org/10.1590/S1516-18462008000400010

Belafsky, P., Blumenfeld, L., LePage, A., \& Nahrstedt, K. (Nov de 2003). The accuracy of the modified Evan's Blue Dye Test in predigting aspiration. Laryngoscope, 113(11), 1969-72. doi:https://doi. org/10.1097/00005537-200311000-00021

Simao, M., Alacid, C., Rordrigues, K., Albuquerque, C, \& Furkim, A. (Oct-Dec de 2009). Incidence of tracheal aspiration in tracheotomized patients in use of mechanical ventilation. Arquivos de gastroenterologia, 46(4), 311-314. doi: https://doi.org/10.1590/S0004-28032009000400012

Fiorelly, A., Fausto, F., Nagar, F., Fusco, P., Mazzone, S., Costa, G., . . Santini, M. (31 de abril de 2017). A New Modified Evans Blue Dye Test as Screening Test for Aspiration in Tracheostomized Patients. Journal of cardiothoracic and vascular anesthesia., 2, 441-445. doi:https://doi. org/10.1053/j.jvca.2016.07.031

O’Neil, K., Purdy, M., Falk, J., \& Gallo, L. (1999). The dysphagia outcome and severity scale. Dysphagia., 14(3), 139-145. doi:https://doi.org/10.1007/ PL00009595

Rosenbek, J., Robbins, J., Roecker, E., Coyle, J., \&Wood, J. (1996). A penetration-aspiration scale. Dysphagia, 11(2), 93-98. doi:https://doi. org/10.1007/bf00417897

Guevara, E., Avendaño, S., Salazar, A., Alarcón, M., \& Santelices, M. (2016). Contribución de la temperatura fría y el sabor ácido en la intervención fonoaudiológica de la disfagia orofaríngea. Revista chilena de fonoaudiología, 15, 1-9. doi: https://doi.org/10.5354/07194692.2016.44206

International Dysphagia Diet Standardisation Initiative. (01 de Agosto de 2018). International Dysphagia Diet Standardisation Initiative. Obtenido de IDDSI: http://iddsi.org/

Pereira, G., Carrera, E., \& Brandão. (2010). Principios da reabilitação das disfagias orofaríngeas. En G. Jotz, E. Carrara-De Angelis, \& A. Barros, ratado da deglutição e disfagia No adulto e na criança (págs. 332339). REVINTER.

Notario, M., Arauz, L., Granados, F., \& Marmol, P. (22 de Febrero de 2011). Plan de cuidados en el síndrome de cautiverio. Enfermería Clínica, 21(2), 104-109. doi:https://doi.org/10.1016/i.enfcli.2010.11.005

World Medical Assault Strength. (2013). 64th General Assembly. Declaration of Helsinki of the amm - Ethical Principles for Medical Investigations in Human Beings. Fortaleza: World Medical Association. Obtenido de https://www.wma.net/policies-post/wma-declaration-of-helsinkiethical-principles-for-medical-research-involving-human-subjects/

República de Colombia. Ministerio de Salud. (1993). Resolución Nº 008430. Bogotá: Ministerio de Salud, Rama ejecutiva - República de Colombia.

Costa, M. (2013). Controle Neural da Deglutição. En Deglutição \& Disfagia: Bases Morfofuncionais e Videofluroscópicas (págs. 51-53). Rio de Janeiro: Medbook.

Cola, P., Gatto, A., Gonçalves, R., Schelp, A., \& Coelho, M. (2008). Reabilitação em disfagia orofaríngea neurogênica: sabor azedo e temperatura fría. Rev. CEFAC, 10(2). doi:https://doi.org/10.1590/S1516$\underline{184620080000200009}$

Ney, D., Weiss, J., Kind, A., \& Robbins, J. (2009). Senescent Swallowing: Impact, Strategies and Interventions. Nutr Clin Pract., 24(3), 395-413. doi: https://doi.org/10.1177/0884533609332005
Steele, C., Abdulrahman, W., Ayanikalath, S., Barbon, C., Chen, J., Cichero, J., ... Wang, H. (Octubre de 2015). The Influence of Food Texture and Liquid Consistency Modification on Swallowing Physiology and Function: A Systematic Review. Dysphagia, 30, 2-26. doi: https://doi. org/10.1007/s00455-014-9578-x

Clavé, P., Arreola, V., Romea, M., Medina, L., Palomera, E., \& Serra-Prat, M. (2008). Accuracy of the volume-viscosity swallow test for clinical screeneng of oropharyngeal dysphagia and aspiration. Clinical Nutrition, 2(7), 806-15. doi:https://doi.org/10.1016/j.clnu.2008.06.011

Barros, A., Silva, S., \& De Angelis, E. (2010). Videofluoroscopia da deglutição orofaríngea. En G. Jotz, E. Carrara-De-Angelis, \& A. Barros, Tratado de deglutição e disfagia: no adulto e na criança. (págs. 84-88). Rio de Janeiro: Revinter.

Castillo E, Y. M., Ibarra N, A. M., Víveros, D. C., \& Ujueta, A. (Enero- Junio de 2017). Uso de Audifonos en Adultos Mayores. Factores personales y ambientales involucrados. Revista Areté, 17(1), 15-19.

OMS. (2015). Organizacion Mundial de la Salud. Obtenido de Informe Mundial Sobre el Envejecimiento y la Salud: https://apps.who.int/iris/ bitstream/handle/10665/186466/9789240694873 spa.pdf? sequence=1

Cano, C. A., Borda, M. G., Arciniegas, A. J., \& Parra, J. S. (2014). Problemas de la Audición en el Adulto Mayor, Factores asociaos y calidad de Vida: estudio SABE, Bogotá, Colombia. Bioédica, 4(34), 574-579.

Cañetes, O., \&A, G. (2009). Descripción de factores no audiológicos asociados en adultos mayores del programa de audífonos año 2006, Hospital Padre Hurtado, Santiago. Revista de Otorrinolaringología y cirugía de cabeza y cuello, 69(1), 29-36.

Calvache-Mora, C. A. (2015). Perspectiva sistémica de la comunicación humana y sus desórdenes. ". Revista Ciencias de la Salud, 13(3), 327329.

Arango, M. Z. (2016). Los axiomas de la comunicación humana en Paul Watzlawick, Janet Beavin, Don Jackson y su relación con la Terapia Familiar Sistémica. Fundacion Universitaria Luis Amigo. 3(1), 33-50.

Vera-García, M. d., López-Tobar, F. R., Valle-Delgado, V. M., \& MazacónGómez, M. N. (Diciembre de 2017). Habilidades Sociales. Revista Salud y Ciencias, 1(2).

Organización Mundial de la Salud. (2001). CIF. Obtenido de Clasificacion Internacional del Funcionamiento, de la Discapacidad y la salud. https://aspace.org/assets/uploads/publicaciones/e74e4-cif 2001.pdf

Bernal-Rodríguez, S. G., Pereira-Alba, O. L., \& Rodríguez-Jiménez, G. E. (2018). Comunicación Humana Interpersonal. Una mirada sistémica. Bogotá, Colombia: IberAM, Corporaciín Universitaria Iberoamericana.

Real Academia Española. (2001). Diccionario de la lengua Española. España.

Zavala G, M. e. (2006). Funcionamiento Social del adulto Mayor. Ciencia y enfermería, 12(2), 53-62.

Bustamante, M., Vidal, C., \& Leopoldo, L. (2014). Impacto del Uso de Audífonos para Adultos Mayores en Chile. Inf. Tecnol, 25(3), 177-184.

Leon I, A., Rodrigo, E. R., \& carvallo, R. (2010). Adherencia al uso de los audifono en adultos mayores del servicio de salud Aconcagua. Rev. Otorrinolaringología. Cir. Cabeza y Cuello, 70(1), 37-42.

Salazar. (2015). Habilidades Sociocomunicativas en base a los interlocutores disponibles en el adulto mayor sano. Revista Chilena de Neuropsicologia. 10(1)., 1-4.

León I. (2010). Adherencia al uso de audífonos en adultos mayores del servicio de Aconcagua. Revista Otorrinolaringología Cirugía cabeza y cuello. Online (70), 37-42.

Cardemil, F. (2016). Adherencia al uso de audífonos en adultos mayores con hipoacusia: Un Ensayo Clínico Aleatorizado y Consideraciones para la definición programática. Chile.

Bustamante, M. A. (2014). Impacto del Uso de Audífonos para Adultos Mayores en Chile. Informacion Tecnologica, 3(25), 177-184.

Castillo, Y. M. (2017). Uso de Audifonos en Adultos Mayores: Factores personales y ambientales involucrados. Revista Arete., (1)(17), 15-19. 
Rodríguez Hernández, Y. (2009). La competencia comunicativa adulta producto de un complejo mundo de saberes y experiencias. Arete 9 (1)., 6-10.

Zavala M., V. C. (2006). Funcionamiento Social del Adulto Mayor. . Ciencia y Enfermeria XII (2), 53-62.

Bruner , J. (1981). De la comunicación al lenguaje: Una perspectiva psicológica. . Infancia y Aprendizaje. 4 (sup1). , 133-163. Obtenido de doi: https://doi.org/10.1080/02103702.1981.10821894

Halliday, M. (1973). Explorations in the functions of language, 25 Hill Street. Londres. WIX 8LL. Inglaterra.: Edward Arnold (Publishers) Ltd.

Buhler, K. (1990). Theory of Language: The Representational Function of Language. Philadelphia, Pennsylvania, U.S.A. : Publicado por John Benjamins Publishing Co.

Warrillow, S., Farley, K., \& Jones, D. (2015). Ten practical strategies for effective communication with relatives of ICU patients. Intensive Care Medicine, 41(12), 2173-2176. Obtenido de https://doi.org/10.1007/ s00134-015-3815-0

Grossbach, I., Stranberg, S., \& Chlan, L. (2011). Promoting Effective Communication for Patients Receiving Mechanical Ventilation. Critical Care Nurse, 31(3), 46-61. Obtenido de https://doi.org/10.4037/ ccn2010728

Ortega-Chacón, V., \& Martínez Díaz, D. (2017). Estrategias e instrumentos de comunicación con pacientes en ventilación mecánica invasiva. Revisión de la literatura. Revista Española de comunicación en Salud, 8(1), 92-100. Obtenido de https://doi.org/10.20318/recs.2017.3608

Goncalves, M. (2008). O significado da comunicação no atendimento ao paciente em UTI: ¿Cómo o fonoaudiólogo pode ajudar? Mundo Saúde. 32(1): 79-84, jan.-mar.

Broyles, L., Tate, J., \& Happ, M. (2012). Use of augmentative and alternative communication strategies by family members in the intensive care unit. Am J Crit Care. Mar. 21(2): e21-32. doi: https://doi.org/10.4037/ ajcc2012752. PMID: 22381993 [PubMed - indexed for MEDLI.

Ijssennagger, C. E., Diez Hoorn, S., Girbes, A. R., \& Tuinman, P. R. (2017). A new speech enhancement device for critically ill patients with communication problems: a prospective feasibility study. Intensive Care Medicine, 43(3), 460-462. Obtenido de https://doi.org/10.1007/ s00134-016-4629-4.

Rodríguez, C., \& Rowe, M. (2010). Use of a speech-generating device for hospitalized postoperative patients with head and neck cancer experiencing speechlessness. Oncology Nursing Forum. 37(2), 199205. Recuperado de: $h$ ttps://doi.org/10.1188/10.0NF.199-20. Obtenido de https://doi.org/10.1188/10.0NF.199-205.

Aranda Hernández, B., \& Saez Ruiz, I. (2017). Relación de ayuda de enfermería a pacientes y familiares en la unidad de cuidados intensivos. Revista Española de comunicación En Salud, 8(2), 239_ 246. Obtenido de https://doi.org/10.20318/recs.2017.4004

Baddley, D. (2018). Enhancing Effective Communication Among Non-Verbal Patients. Pediatric Nursing. 44(3). 144-146. Obtenido de http://search. ebscohost.com/login.aspx?direct=true\&db=tfh\&AN=130397368\&lang=e s\&site=ehost-live

Dithole, K. S., Thupayagale-Tshweneagae, G., Akpor, O., \& Moleki, M. (2017). Communication skills intervention: promoting effective communication between nurses and mechanically ventilated patients. Dec 15;16;74. Obtenido de doi: https://doi.org/10.1186/ s12912-017-0268-5. eCollection 2017.

Benbenishty, J. S., \& Hannink, J. R. (2015). Non-verbal communication to restore patient-provider trust. Intensive Care Medicine. 41(7). 13591360. Obtenido de https://doi.org/10.1007/s00134-015-3710-8

Zaga, C. J., Berney, S., \& Vogel, A. P. (2019). The Feasibility, Utility, and Safety of Communication Interventions With Mechanically Ventilated Intensive Care Unit Patients: A Systematic Review. American Journal of Speech-Language Pathology, 28.1335-1355. Obtenido de https:// doi.org/10.1044/2019_AJSLP-19-0001.
Rojas , N. P., Bustamante-Troncoso , C. R., \& Dois-Castellón, A. (2014). Comunicación entre equipo de enfermería y pacientes con ventilación mecánica invasiva en una unidad de paciente crítico. Aquichan; 14(2): 184-195.

Diez, H. S. (2016). Communicating with conscious and mechanically ventilated critically ill patients: a systematic review. Crit Care. 2016 Oct 19;20(1):333.

Nilsen, M., Sereika, S. M., Hoffman, L. A., Barnato, A., Donovan, H., \& Happ, M. B. (2014). Nurse and patient interaction behaviors' effects on nursing care quality for mechanically ventilated older adults in the ICU. . Obtenido de Res Gerontol Nurs. May-Jun;7(3):113-25. doi: https://doi.org/10.3928/19404921-20140127-02. Epub 2014. Feb 5. PMID: 24496114. [PubMed - indexed for MEDLINE].

Hosseini, S. R., Valizad-Hasanloei, M.-A., \& Feizi, A. (2018). El efecto del uso de paneles de comunicación sobre la facilidad de comunicación y la ansiedad en pacientes conscientes con ventilación mecánica ingresados en unidades de cuidados intensivos. . Obtenido de Revista iraní de investigación en enfermería y partería, 23 (5), 358362.: doi: https://doi.org/10.4103/ijnmr.IJNMR_68_17.

Jansson, S., Rivera SanMartín, T., Johnson, E., \& Nilsson, S. (2019). Jansson, S., Martin, TRS., Johnson, E., Nilsson, S. Healthcare professionals use of augmentative and alternative communication in an intensive care unit: A survey study. Intensive Crit Care Nurs. Apr 17. pii: S0964-3397(18)30365-3. . Obtenido de https://doi.org/10.1016/j. iccn.2019.04.002

Rojas Silva, N., Amthauer, M., \& Bustamente Troncoso, C. (2014). Comunicación con el paciente en ventilación mecánica invasiva: Revisión de la literatura. Revista Chilena de Medicina Intensiva 2014; Vol 29(2): 115-119.

Radtke, J., Baumann, B., Garrett, K., \& Happ, M. (2011). Listening to the Voiceless Patient: Case Reports in Assisted Communication in the Intensive Care. Unit. Journal of Palliative Medicine, 14(6), 791-795. Obtenido de Recuperado de: https://doi.org/10.1089/jpm.2010.0313

Radtke, J., Tate, J., \& Happ, M. (2012). Nurses' perceptions of communication training in the ICU. Intensive Crit Care Nurs. Feb;28(1):16-25. doi: https://doi.org/10.1016/j.iccn.2011.11.005. Epub 2011 Dec 14. PMID: 22172745 [PubMed - indexed for MEDLINE].

Momennasab, M., Shaker Ardakani, M., Dehghan Rad, F., Dokoohaki, R., Dakhesh, R., \& Jaberi, A. (2019). Momennasab, M., Ardakani, M. S., Rad, F. D., Dokoohaki, R., Dakhesh, R., y Jaberi, A. (2019). Quality of Nurses' Communication with Mechanically Ventilated Patients in a Cardiac Surgery Intensive Care Unit. Investigacion \& Educación en enfermería, 37(2),. Obtenido de https://doi.org/10.17533/udea.iee. v37n2e02

Gómez.Carretero, P., Monsalve, V., Soriano, J., \& de Andrés, J. (2007). Alteraciones emocionales y necesidades psicológicas de pacientes en una Unidad de Cuidados Intensivos. Obtenido de http://scielo.isciii. es/scielo.php?script=sci arttext\&pid=S0210-56912007000600006

Vargas, R. (2007). Cuidado humanizado al paciente críticamente enfermo: Enfermería, pieza clave en la atención. Ciencia y cuidado. Volumen 4.No. 4. ISSN- 17949831.

Castaño, S. I., Segura, M. C., Molina, R., Corredor, N., Neira, L., Mambrú, M., .. Castellano, J. (2014). Perfil y competencias del fonoaudiologo en Colombia. Obtenido de https://www. minsalud.gov.co/sites/rid/Lists/ BibliotecaDigital/RIDE/VS/TH/Fonoaudiologia Octubre 2014.pdf: https://www.minsalud.gov.co/sites/rid/Lists/BibliotecaDigital/RIDE/VS/ TH/Fonoaudiologia Octubre 2014.pdf

Tracy, M. F., \& Chlan, L. (2011). Nonpharmacological Interventions to Manage Common Symptoms in Patients Receiving Mechanical Ventilation. Critical Care Nurse, 31(3), 19-29. Obtenido de https://doi. org/10.4037/ccn2011653

Happ, M. B., Garrett., K. L., Tate, J. A., DiVirgilio, D., Houze, M. P., Demirci, J., ... Sereika, S. M. (2014). Effect of a multi-level intervention on nurse-patient communication in the intensive care unit: results of the SPEACS trial. Heart Lung. M. 
Gropp, M., Johnson, E., Bornman, J., \& Koul, R. (2019). Nurses’ perspectives about communication with patients in an intensive care setting using a communication board: A pilot study. Health SA Gesondheid, 24. 9 pages. Obtenido de https://doi.org/10.4102/hsag.v24i0.1162

Sáez Ruiz, I. M., \& Aranda Hernández, B. (2017). Relación de ayuda de enfermería a pacientes y familiares en la unidad de cuidados intensivos. Revista Española de comunicación En Salud, 8(2), 239246. . Obtenido de https://doi.org/10.20318/recs.2017.4004

Ronda, J. M. (2002). Asociación entre síntomas clínicos y resultados de la posturografía computarizada dinámica. Acta Otorrinolaringológica Española. Vol. 53(4), 252-255.

R., G. (2007). Relación entre los sistemas vestibulares, visual y auditivo. Univ Play Ancha. Vol. 13.

Civantos, C. C. (2018). Primeros pasos en la posturografía dinámica computarizada. Revista ORL. Vol. 9(3), 227-237.

Cole, E. B. (2015). Children with hearing loss: Developing listening and talking, birth to six. San Diego, CA: Plural Publishing.

Cardoso, C. C. (2018). Development of postural control and maturation of sensory systems in children of different ages a cross-sectional study. Brazilian Journal of Physical Therapy. Vol. 22(1), 70-76.

Daley, M. L. (1982). Quantitative Posturography: Use in Multiple Sclerosis. IEEE Transactions on Biomedical Engineering, BME. Vol. 28(9), 668671.

Gouléme, e. a. (2018). Changes of spatial and temporal characteristics of dynamic postural control in children with typical neurodevelopment with age: Results of a multicenter pediatric study. International Journal of Pediatric Otorhinolaryngology. Vol. 113, 272-280.

Reyes, A. S.-T. (2018). Computerised Dynamic Posturography in Premanifest and Manifest individuals with Huntington's Disease. Scientific reports. Vol. 8(1), 14615.

Bujang, R. W. (2013). Posture Stability in Adult Cochlear Implant Recipients. Journal of Medical Sciences. Vol. 13, 86-94.

Andrew Kelly, Z. L. (2018). Balance in children following cochlear implantation. Cochlear Implants International. Vol. 19(1), 22-25.

Licameli G, Z. G. (2009). Disturbance of vestibular function attributable to cochlear implantation in children. Laryngoscope. Vol.19(4), 704-745.

Ramírez, A. G. (2016). Análisis clínico y posturográfico del equilibrio en ancianos caedores de repetición. Universidad Complutense de Madrid, 1.
Bascuas Rodríguez, I. \&. (2013). Cambios en el equilibrio medido mediante posturografía computerizada al año de una prótesis de rodilla.

Del Moral Orro, G. M. (2013). Del marco teórico de integración sensorial al modelo clínico de intervención. Revista electrónica de terapia ocupacional Galicia, TOG. Vol. 17, 18.

ASHA. (2019). ASHA.org. Obtenido de Hearing Loss (Ages 5+): Overview. : https://www.asha.org/PRPSpecificTopic. aspx?folderid $=8589935335 \&$ section $=$ Overview

Bisdorff, A. S.-T. (2015). Resumen de la Clasificación Internacional de Trastornos Vestibulares. Neurol Clin. Vol. 33, 546-547.

Díaz, C. G. (2016). Hipoacusia: Trascendencia, incidencia y prevalencia. Revista Médica Clínica Las Condes. Vol. 27(6), 732.

Kelly, A. L. (2018). Balance in children following cochlear implantation. Cochlear implants international, Vol.19(1), 22-25.

Lane, S. J. (2013). Sensory integration and processing: Theory and applications to occupational performance. En G. G. B. A. Boyt Schell, Willard and Spackman's occupational therapy (12th ed) (págs. 816-868). Philadelphia: Lippincott Williams \& Wilkins.

León, J. B. (2014). Vértigo periférico frente a vértigo central. Aplicación del protocolo HINTS. Revista de neurología. Vol. 59(8), 349-353.

Marulanda Ruiz MA, T. A. (2017). Caracterización del estado auditivo del personal del batallón de mantenimiento de aviones $n^{\circ} 1$ división de aviación ejército nacional. .

Nandi, R. \&. (2008). Development and assessment of the vestibular system. International Journal of Audiology. Vol. (47), 566-577.

National Institute on Deafness and Other Communication Disorders. (2017). Implantes Cocleares. Obtenido de https://www.nidcd.nih.gov/es/ espanol/implantes-cocleares

Núñez-Batalla, F. J.-C.-C.-A.-U.-F. (2017). Diagnóstico etiológico de la sordera infantil: recomendaciones de la CODEPEH. Acta Otorrinolaringológica Española; Vol. 68(1), 43-55.

Sokolov, M. G. (2019). Vestibular and balance function is often impaired in children with profound unilateral sensorineural hearing loss. Hearing research. Vol. 372, 52-61.

Tudela-Torras M, A.-M. L.-T. (2017). Integración sensorial: beneficios y efectividad del abordaje terapéutico en los trastornos del procesamiento sensorial. Rev Neurol. Vol. 64(1), 3-7.

Valente, M. (2011). Assessment Techniques for Vestibular Evaluation in Pediatric Patients. Otolaryngol Clin. Vol. 44, 287-288. 